\title{
Zu den Briefen des Theodoros Laskaris.")
}

Die vom italienischen Gelehrten mit wahrer Selbstentsagung and warmer Hingebung herausgegebenen Briefe sind von grolser Bedeutung für die Charakteristik sowohl der eigenartigen Person des Kaisers als auch der interessanten politischen und kulturellen Geschichte seiner Regierungszeit (s. Krumb., Byz. Litteraturgesch. S. 478): dies hat uns Heisenberg, Byz. Z. IX S. 211-221, in einer geschickten Übersicht klar gemacht. Wie verhält es sich nun aber mit dem Zustande der handschriftlichen Überlieferung der Briefe und mit der kritischen Gestaltung der Texte? Zwar hat Festa für die Verbesserung korrupter Stellen vieles geleistet, auch steuerten Heisenberg und Treu (in den Addenda) nicht weniges bei; das meiste ist aber nach meiner festen Überzeugung noch zu thun (die Kritik von A. Papadopulos-Kerameus, Vizant. Vremennik VI, 1899, S. 551 f., hat die Sache nur wenig gefördert), und dies will meine vorliegende spezielle Besprechung auch den Lesern der Byz. Z. in extenso beweisen. Sie beruht auf einer eingehend und wiederholt durchgeführten peinlichen kritischen Untersuchung (die Lektüre der Theodoros-Produkte ist eben nicht sehr amüsant), und das Resultat ist, dafs es mit einer von einer strengen Festhaltung der kritischen Grundsätze ausgehenden zweiten Ausgabe weit anders aussehen würde.

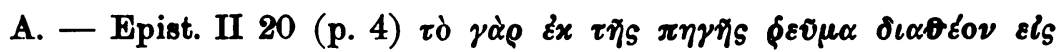

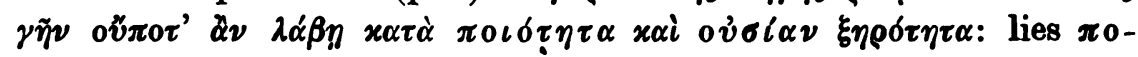

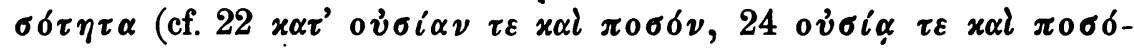

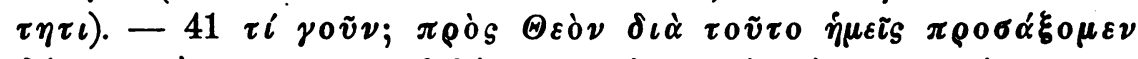

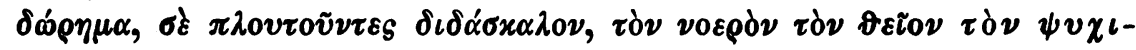

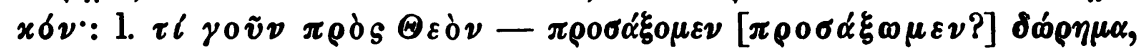

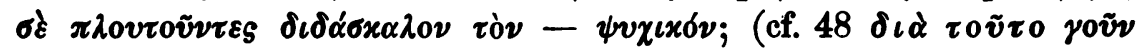

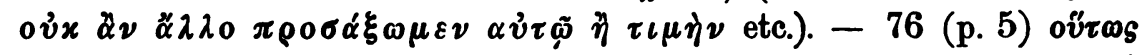

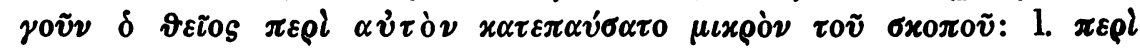
$\alpha \dot{v} \tau \tilde{\omega} \nu$ (betreffs dieser Angelegenheiten).

1) Theodori Ducae Lascaris epistulae CCXVII. Nunc primum edidit Nicolsus Festa, Firenze 1898: 


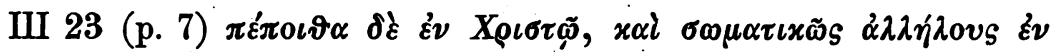

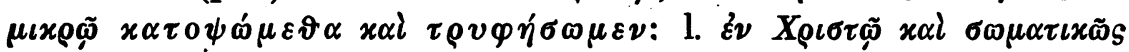

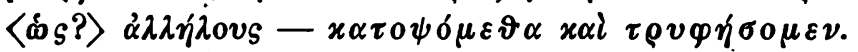

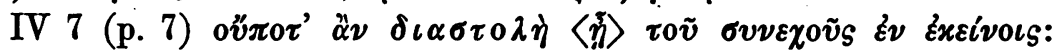
ich habe $\eta^{\xi}$ ergänzt.

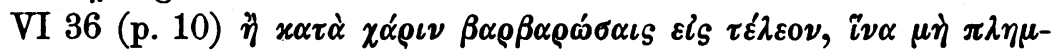

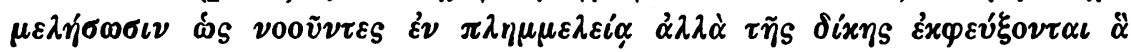

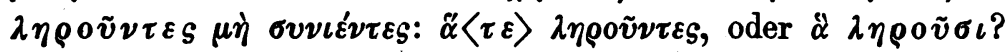

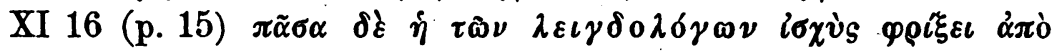

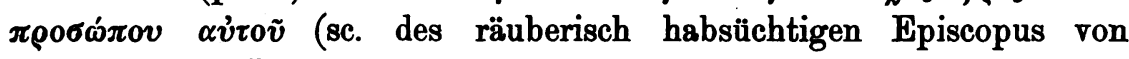

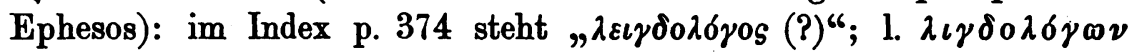
und s. über das Wort $\lambda i \gamma \delta \alpha$ Korais " $A \tau \alpha \kappa \tau \alpha$ I p. 264. Was Theodoros

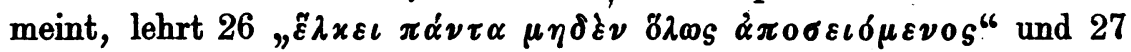

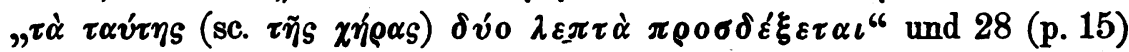

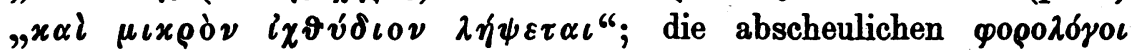
werden vom Kaiser sarkastisch $\lambda \iota \gamma \delta 0 \lambda \delta$ jo

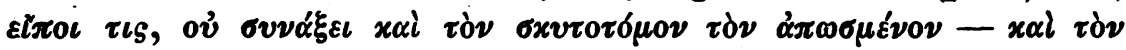

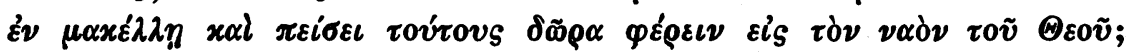

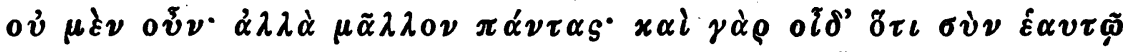

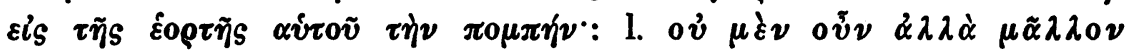

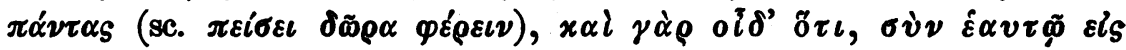

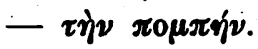

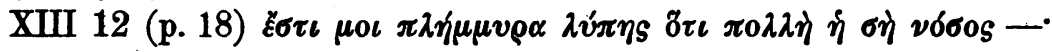

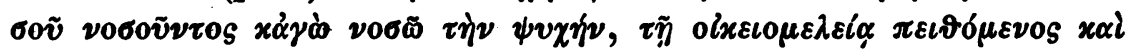

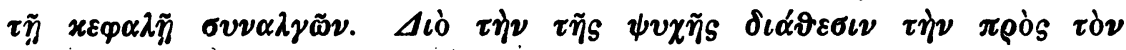

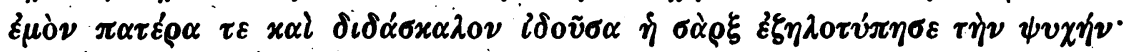

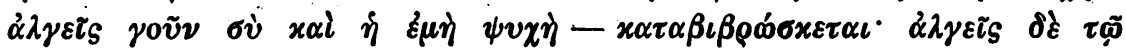

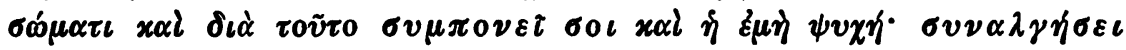

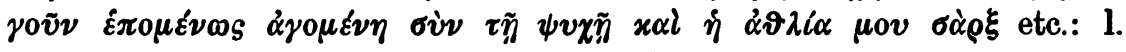

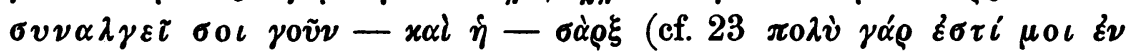

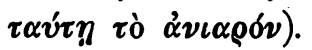

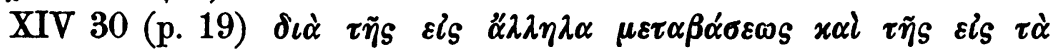

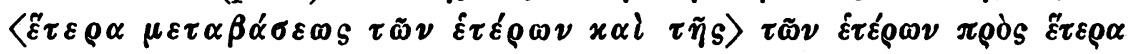

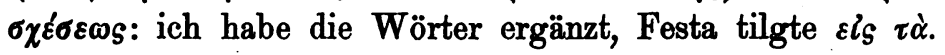

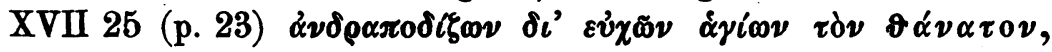

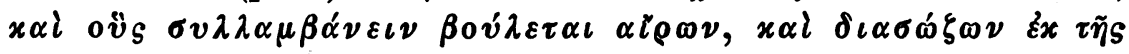

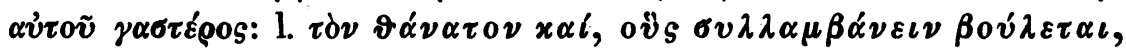
$\alpha \iota \rho \omega \nu x \alpha i \delta \iota \alpha \sigma \omega \dot{\xi} \omega \nu$.

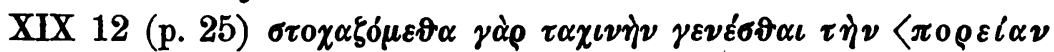

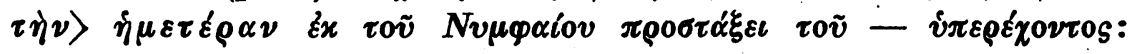

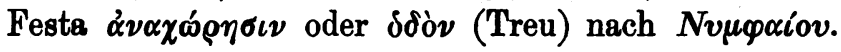

Byzant. Zeitschrift XI 1 u. 2. 


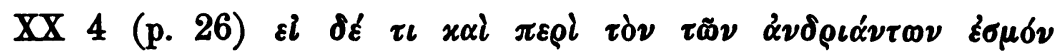

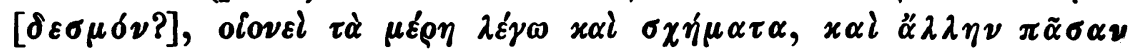

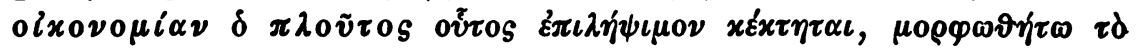

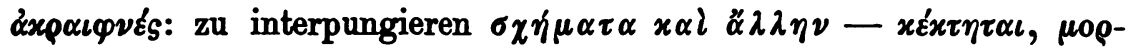
$\varphi \omega \vartheta \boldsymbol{r} \tilde{\tau} \boldsymbol{i}$ etc.

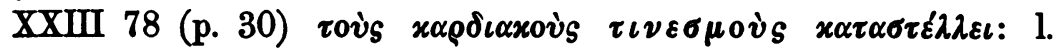
$\tau \varepsilon \iota \nu \varepsilon \sigma \mu o \dot{v} s$ (Festa $\tau \iota \nu \alpha \gamma \mu o \grave{v} \varsigma$ ).

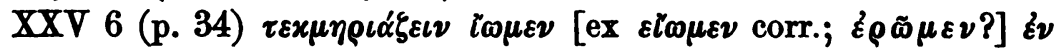

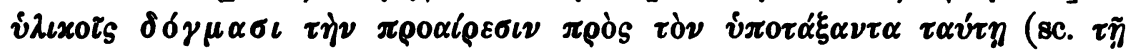

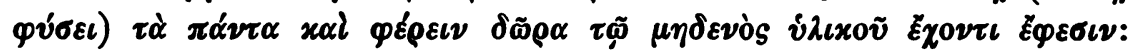
1. $\delta \delta \mu \alpha \sigma \iota$.

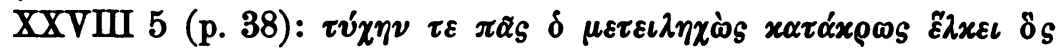

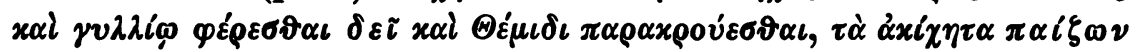

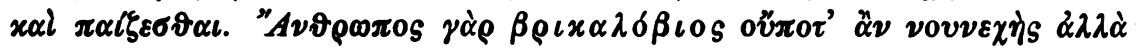

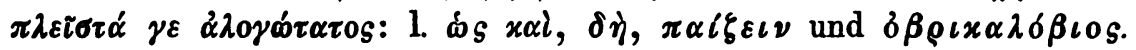

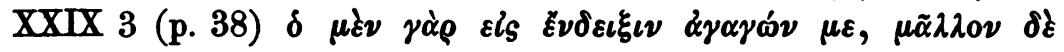

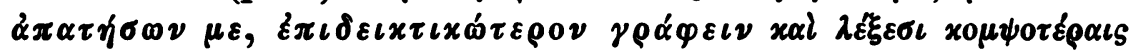

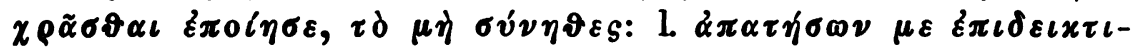

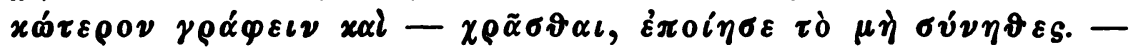

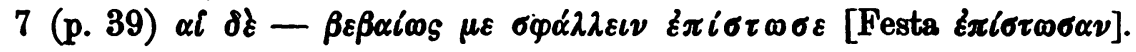

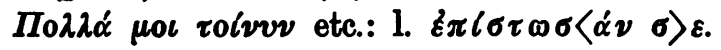

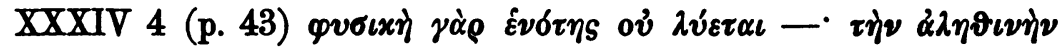

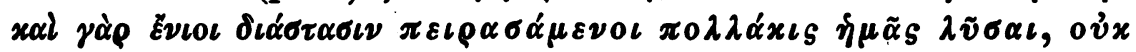

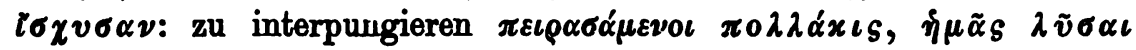
oủx $\iota_{\sigma} \chi v \sigma \alpha \nu$.

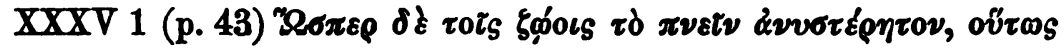

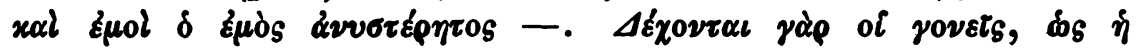

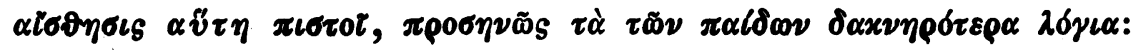
1. $\delta \eta(?)$ und $\alpha \dot{\tau} \tau \dot{\eta}$.

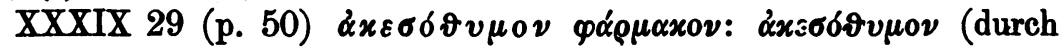
falsche Analogie nach $\alpha x \varepsilon \sigma \omega ́ \delta v \nu 0 \nu$ gebildet?) war nicht in $\alpha x \varepsilon \sigma \zeta \theta v \mu o \nu$

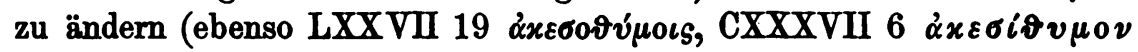

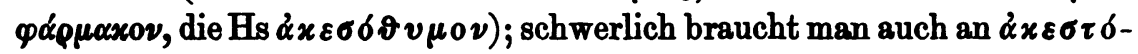
$\vartheta v \mu 0 \nu-\alpha x \varepsilon \sigma \tau 0 \vartheta v \mu o \iota s$ zu denken. - 59 (p. 51) $\tau l_{S} \varepsilon \sigma \tau \iota \nu \delta \alpha \dot{\alpha} \alpha-$

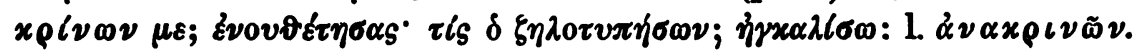

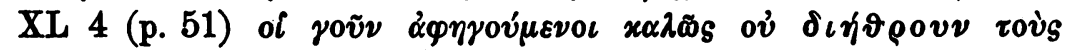

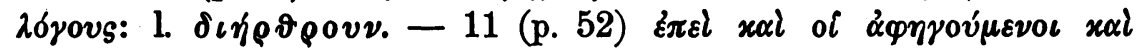

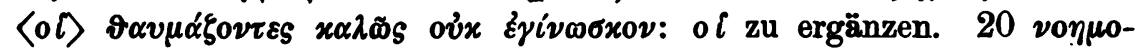

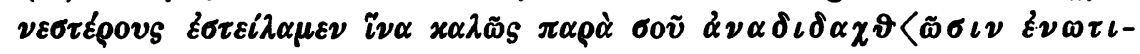

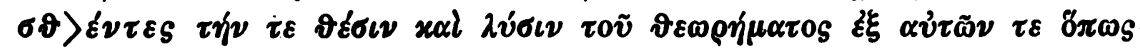

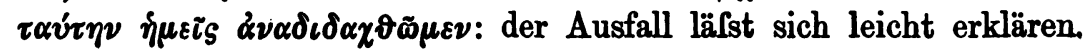




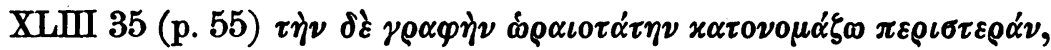

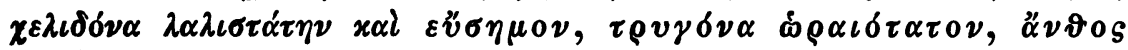

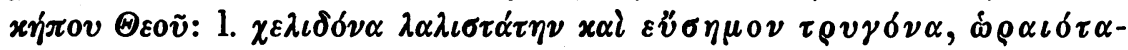

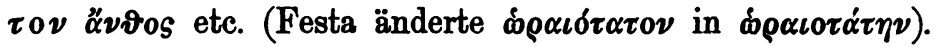

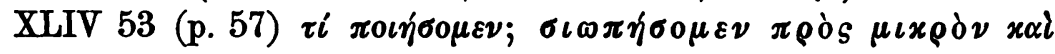

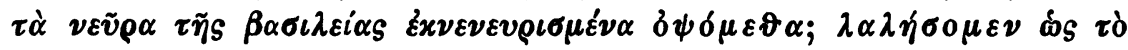

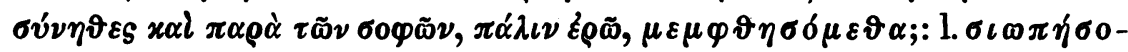

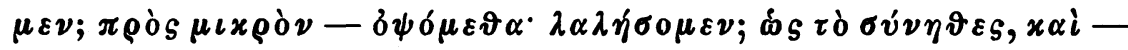

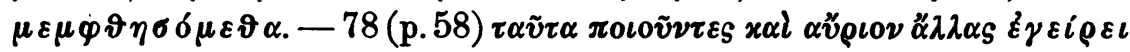
$\mu \varepsilon v$ [deletum] $\mu \varepsilon \rho i \mu \nu \alpha s: 1 . \quad \varepsilon r \varepsilon i \rho o \mu \varepsilon v$.

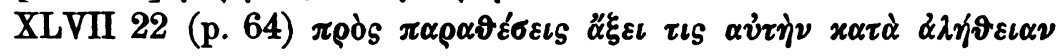

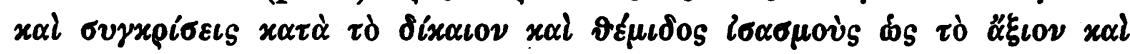

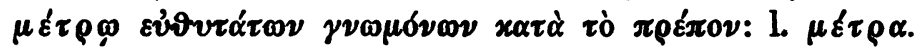

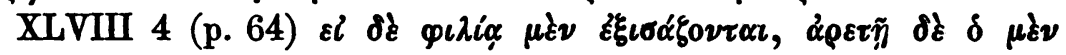

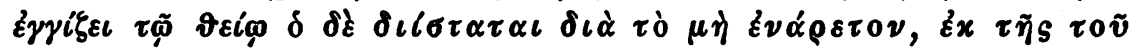

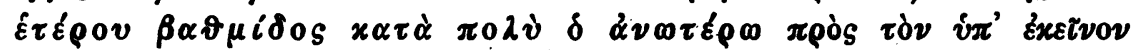

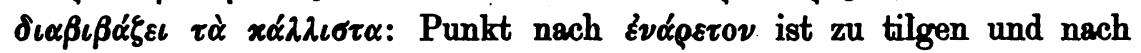

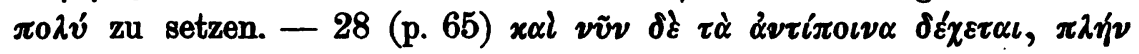

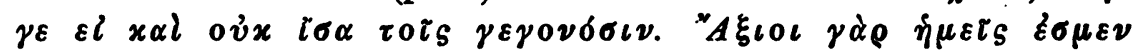

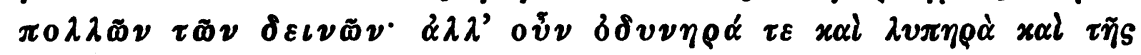

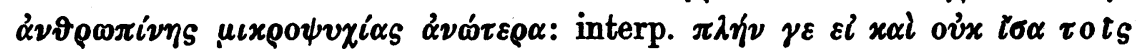
$\gamma \varepsilon \gamma \sigma \nu \delta \sigma \iota \nu(\not \varkappa \xi \iota 0 \iota-\delta \varepsilon \iota \nu \tilde{\omega} \nu) \alpha \lambda \lambda^{\prime} \circ \tilde{v} \nu$ etc. Festa tilgte die W. $\varepsilon l x \alpha i$.

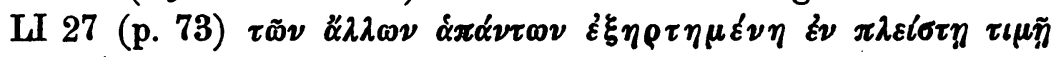

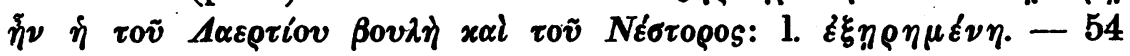

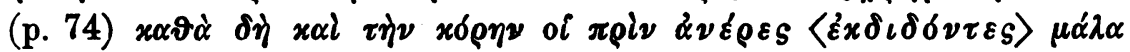

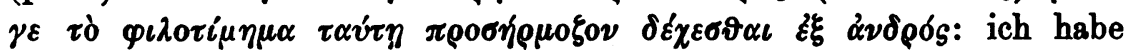
$\varepsilon x \delta \delta \delta \delta v \tau \varepsilon S$ ergänzt (Festa "non intellego"), wenn man nicht vielmehr einen von den abscheulichsten Fällen der kaiserlichen Syntax $(=o i$

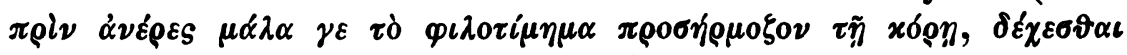

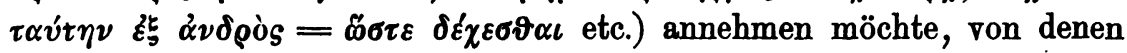

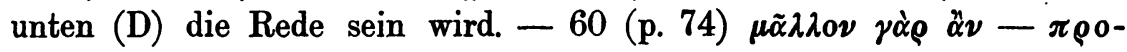

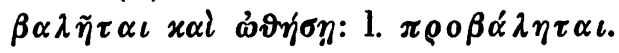

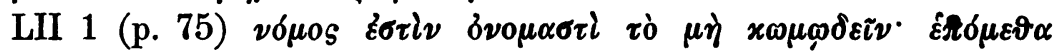

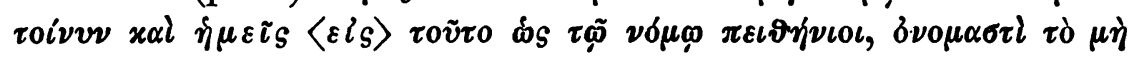

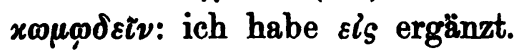

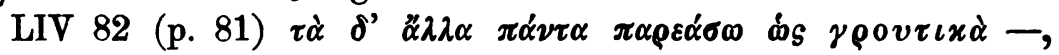

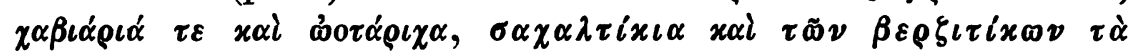

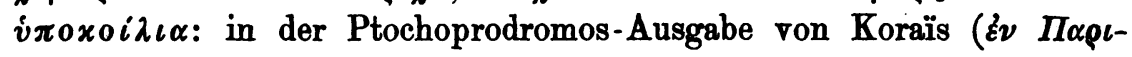

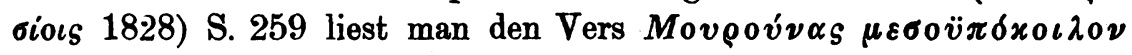
$x \alpha \grave{\alpha} \dot{\pi} \dot{0}$ ì $\sigma \alpha \chi \alpha \lambda \tau i x \iota \nu$, letzteres wohl eine Fischbereitung (Festa Index p. 390 „farcimina, salsicce“, schwerlich richtig); auch $\beta \varepsilon \rho \xi i \tau \iota x o \nu$. 
ist ein Fisch (Koraïs p. 261 und Sophocles Lexicon), $\chi \alpha \beta \iota \alpha$ @ $\iota \alpha$ und

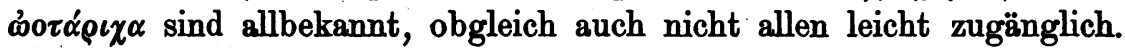

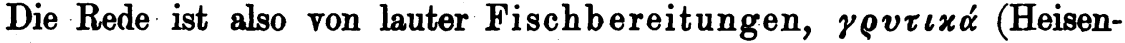

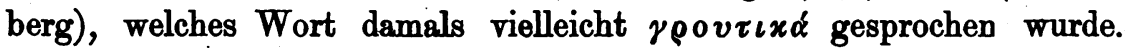
Sonderbar klingt also die im Viz. Vrem. erfundene Zusammenstellung

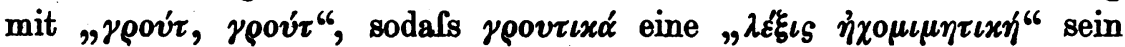

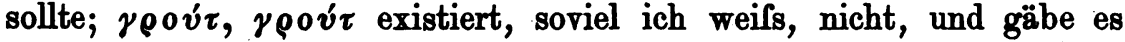
einen solchen $\eta^{\prime} x o s$ ( $\gamma x \circ v \hat{\nu}-\gamma x \lambda$ bei Flüssigkeiten ist bekannt), so würde er sich schwerlich auf Fischspeisen beziehen können, abgesehen

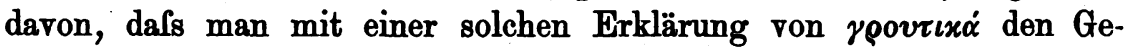

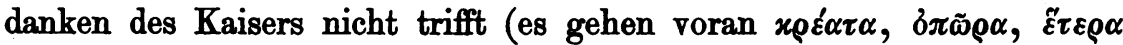

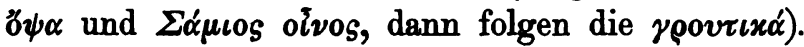

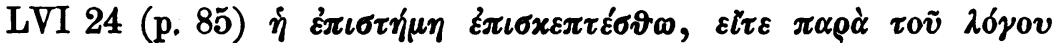

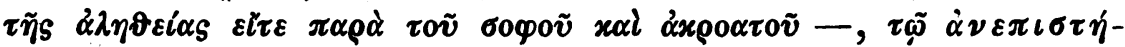

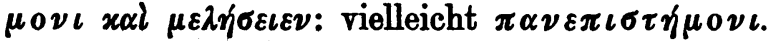

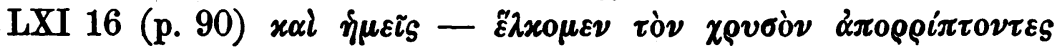

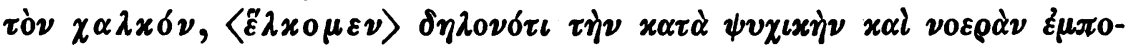

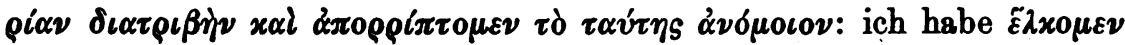

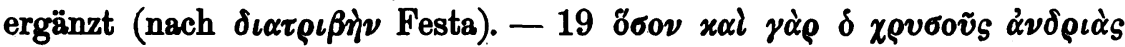

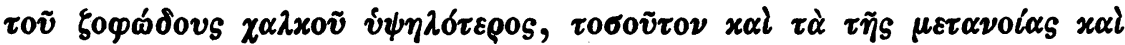

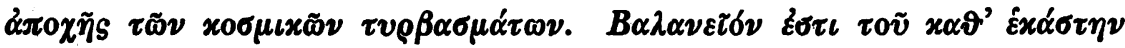
$\delta i \delta \rho \alpha \gamma \mu \alpha \tau \varepsilon \lambda$ ivvros etc.: über $\delta i \delta \rho \alpha \gamma \mu \alpha$ s. die Lexika; Festa $\delta i \delta \rho \alpha \chi \mu \alpha$.

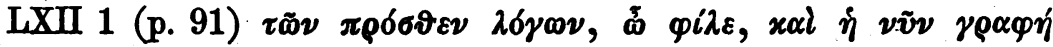

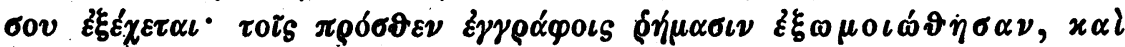

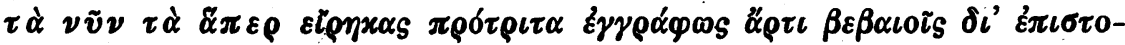

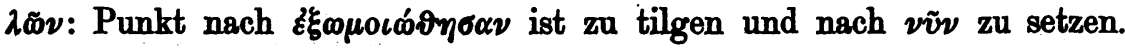

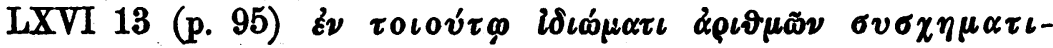
$\sigma \vartheta \tilde{\eta} \nu \alpha \iota:$ vielleicht $\tau 0 \iota 0 v \dot{\tau} \omega \nu$ (cf. 3 Eे $\pi 0 \lambda \lambda \alpha \pi \lambda \alpha \sigma \iota \varepsilon \pi \iota \mu \circ \rho i \omega \nu x \alpha l$

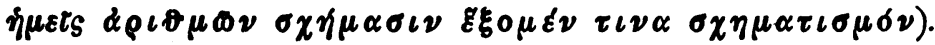

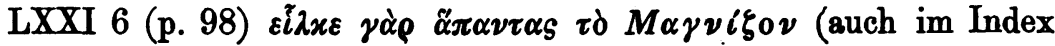

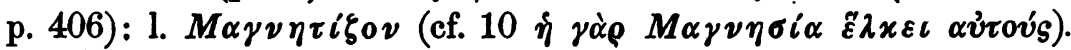

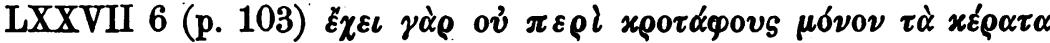

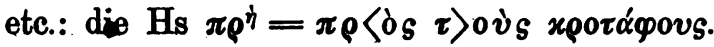

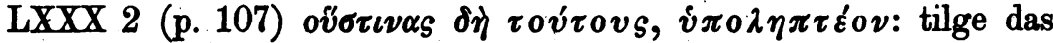

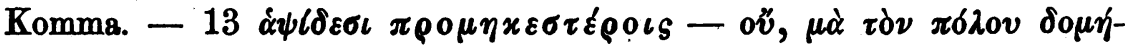

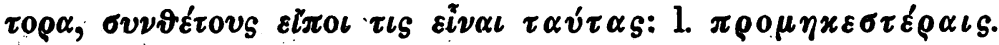

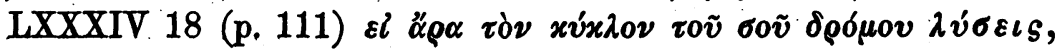

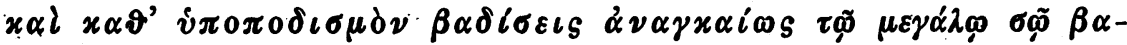

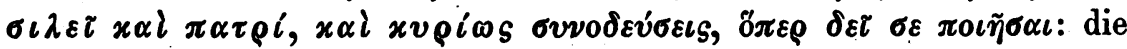

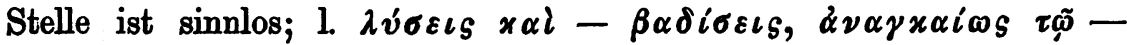

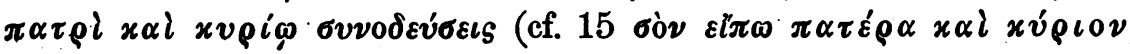




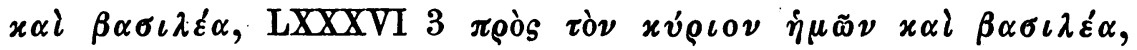

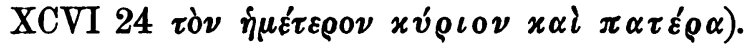

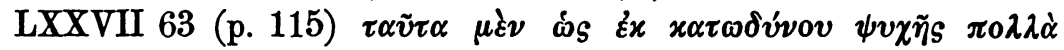

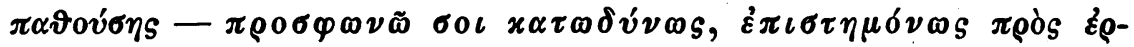

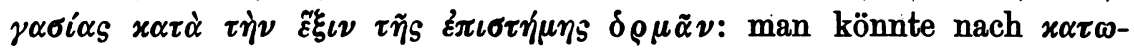
$\delta v^{\nu} \omega s$ das W. $\dot{\omega}_{S}(=\dot{\omega} S-\delta \rho \mu \tilde{\alpha} \nu=\tilde{\omega} \sigma \tau \varepsilon-\delta \rho \mu \tilde{\alpha} \nu)$ oder die W. $\pi \rho \grave{s} \boldsymbol{\tau} \dot{o}(\dot{\varepsilon} \pi l \tau \tilde{w})$ einschieben; die Vermutung ist nicht nötig (ebenso LIV $74 \tau \dot{\eta} \nu-x \lambda \tilde{\eta} \sigma \iota \nu \pi \alpha \rho \varepsilon \dot{\lambda} \varepsilon \iota \psi \alpha s, \mu \eta \delta \xi \mu \nu \eta \sigma \vartheta \tilde{\eta} \nu \alpha \iota \tau \alpha \dot{\tau} \tau \eta S$

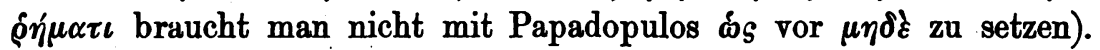

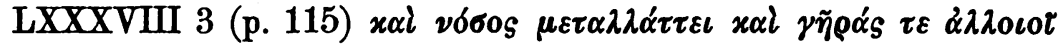

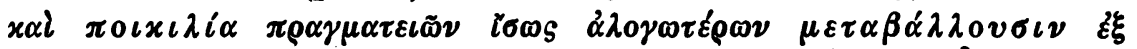
$\alpha \dot{\tau} \tau 0 \tilde{v} \alpha \dot{\tau} \tau \delta \dot{\nu}:$ l. $\pi 0 \iota x \iota \lambda i \alpha \iota$.

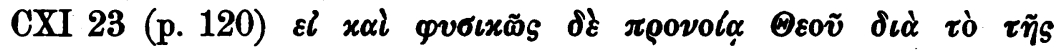

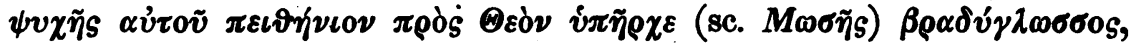

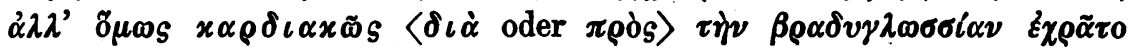

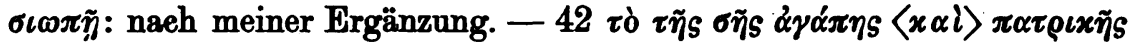

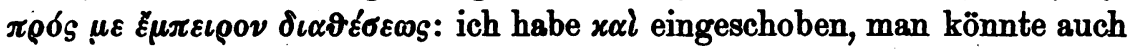

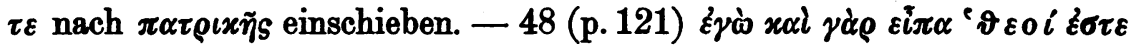

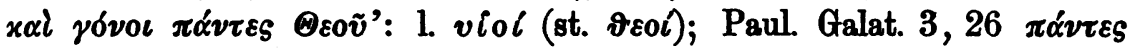

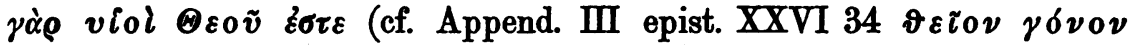

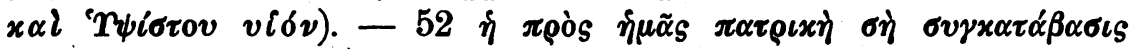
$\varepsilon l x o \nu l \xi \varepsilon \iota \tau \tilde{\eta} \tilde{v} \psi \eta \lambda \tilde{\eta}$ X

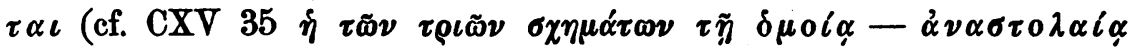

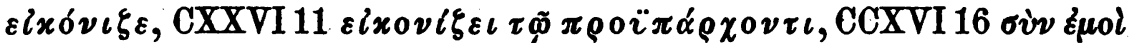

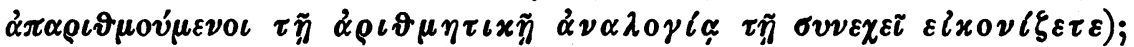
die übrigens gewaltsame Änderung von Papadopulos $\tau \dot{\eta} \nu \dot{v} \psi \eta \lambda \dot{\eta} \nu$

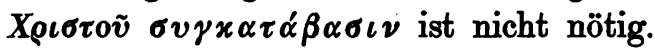

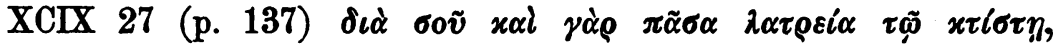

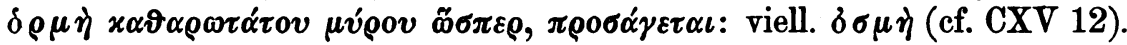

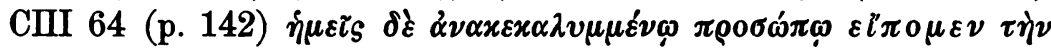

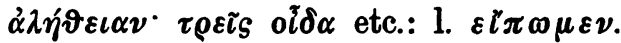

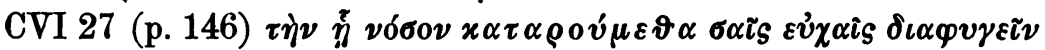
1. $x \alpha \tau \alpha \varrho \gamma \circ v \dot{v} \varepsilon \vartheta \alpha$; man könnte übrigens an $x \alpha \tau \alpha \pi v \varrho o v ́ \mu \varepsilon \vartheta \alpha, x \alpha \tau \alpha-$ $\beta \alpha \varrho \circ v \mu \varepsilon \vartheta \alpha$ (dies mit Kurtz; briefliche Mitteil.), schwerlich an $x \alpha \tau \alpha-$ $\pi \iota \varepsilon \xi \sigma \mu \varepsilon \vartheta \alpha$ (Papadopulos) denken.

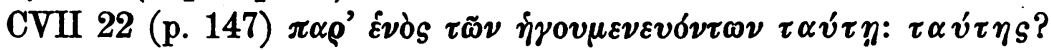

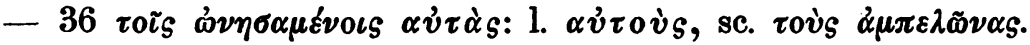

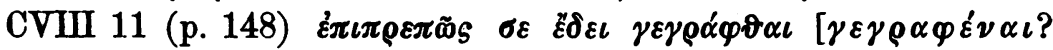

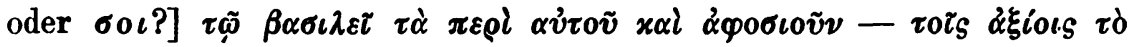

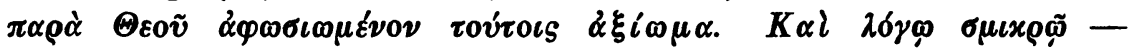

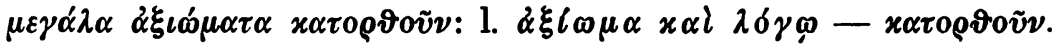




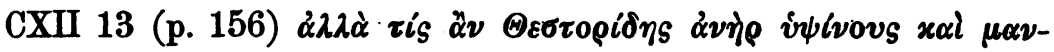

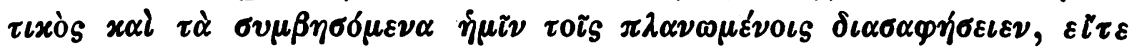

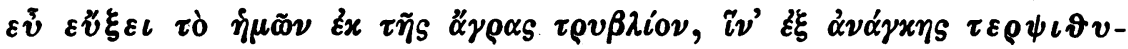

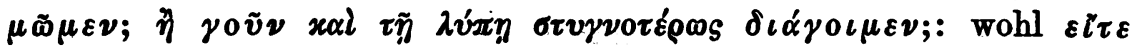

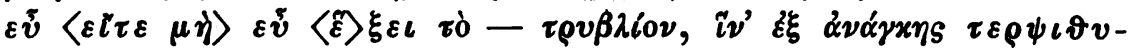

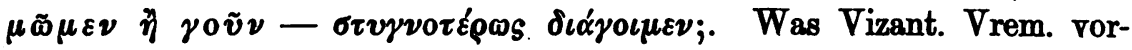
geschlagen wurde $(\varepsilon i \tau \varepsilon \varepsilon \tilde{v} \xi \alpha \iota \tau 0 \quad \eta \mu \tilde{\omega} \nu)$ verstehe ich nicht; keineswegs

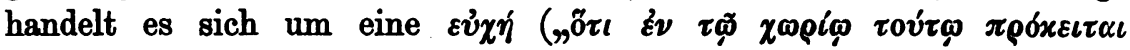

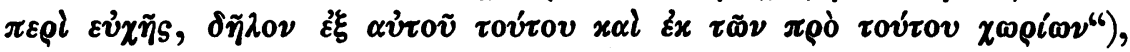
auch ist $\delta\llcorner\alpha ́ \gamma \omega \mu \varepsilon \nu$ nicht nötig (der Wechsel der Tempora ist fast eine Regel).

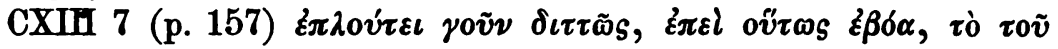

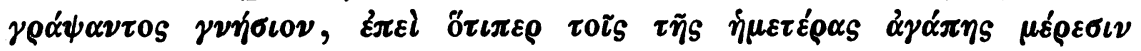

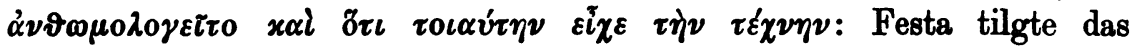
zweite $\varepsilon \pi \varepsilon i$; viell. ist $\dot{\varepsilon} \pi\langle\lambda$ ov́ $\tau\rangle \varepsilon \iota$ zu lesen.

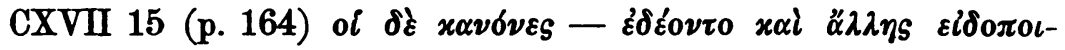

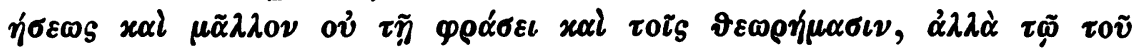

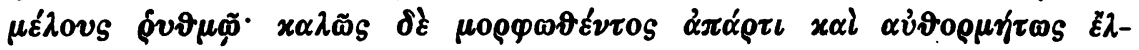

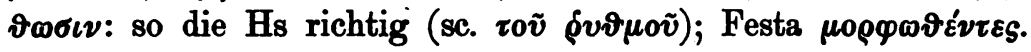

CXVIII 23 (p. 165) $\delta \pi \delta \tau \alpha \nu ~ \delta \xi \delta-\mu \eta \tau \rho о \pi 0 \lambda l \tau \eta S ~ \Sigma \alpha ́ \rho \delta \varepsilon \omega \nu ~ \varepsilon x$

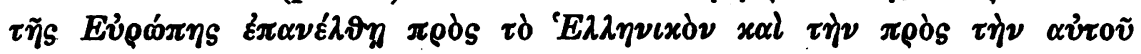

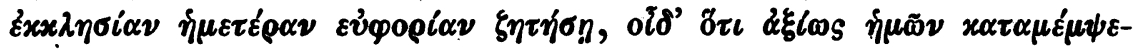

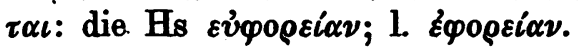

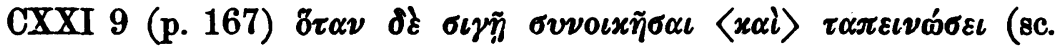

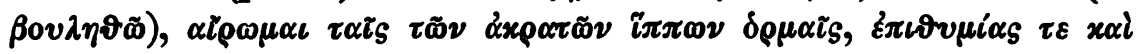

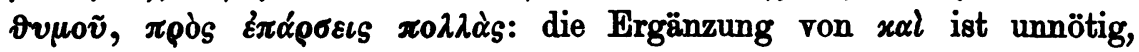
$\sigma \iota \gamma \tilde{\eta} \sigma v \nu 0 \iota x \tilde{\eta} \sigma \alpha \iota=\sigma \iota \gamma \tilde{\eta} \sigma \alpha \iota, \operatorname{dazu} \tau \alpha \pi \varepsilon \iota \nu \omega ́ \sigma \varepsilon \iota$ wie $\operatorname{CXXX} 18 \sigma \iota \omega \pi \tilde{\omega}$ $\tau \tilde{\eta} \tau \alpha \pi \varepsilon \iota \nu \omega ́ \sigma \varepsilon \iota ; 1 . \alpha i \varphi_{\rho} \mu \alpha \iota$.

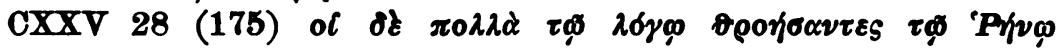

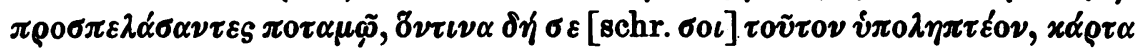

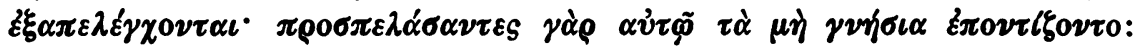

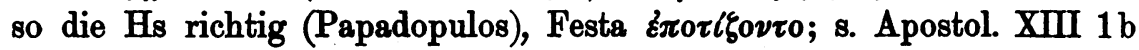

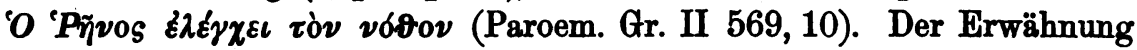
wert ist eine Stelle von Nikephoros, worüber Festa nichts notiert:

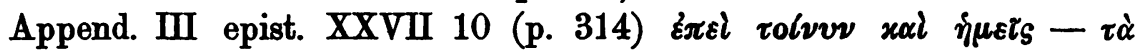

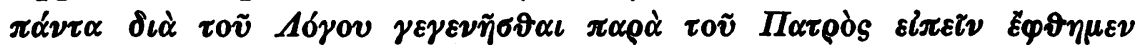

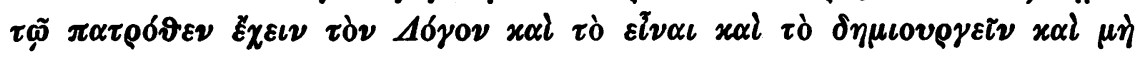

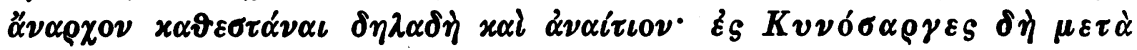

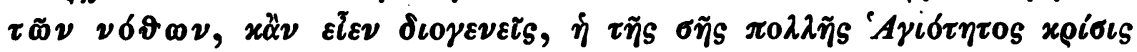

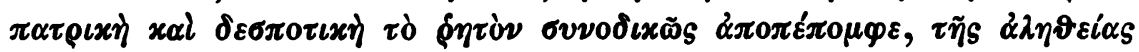

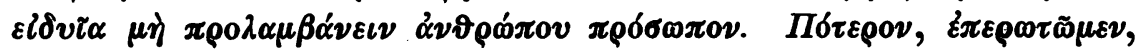




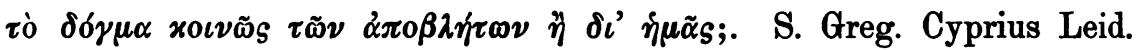

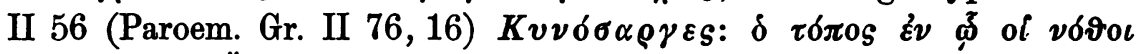

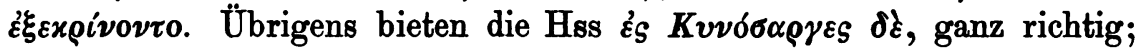

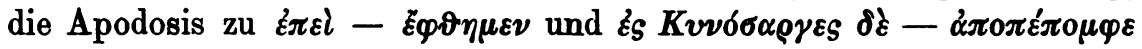

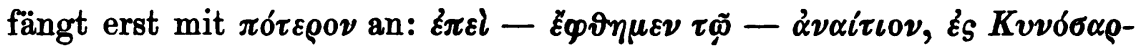

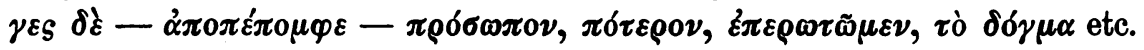
- In dem Briefe von Theodoros bietet die Hs oi $\pi 0 \lambda \lambda \eta^{\prime} \delta \varepsilon$, also oi

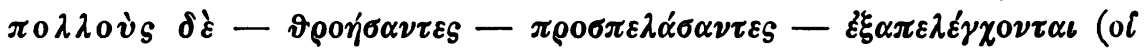
$\delta \varepsilon \pi 0 \lambda \lambda o \dot{v} S-\vartheta \rho o \eta \sigma \alpha \nu \tau \varepsilon S$ Papadopulos).

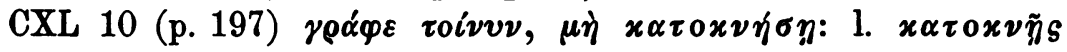
mit der Hs; CLIII $10 \mu \eta \tau \varepsilon \dot{\alpha} \mu \varepsilon \lambda \tilde{\eta} s \quad \mu \eta \tilde{\tau} \varepsilon-x 0 \pi \iota \tilde{\alpha} s$, CXCVIII 28 $\mu \grave{\eta} \pi \alpha \rho \alpha \beta \lambda \varepsilon \dot{\varepsilon} \pi \mathrm{s}$.

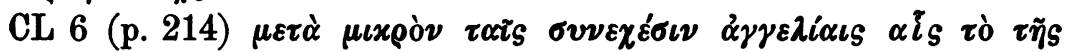

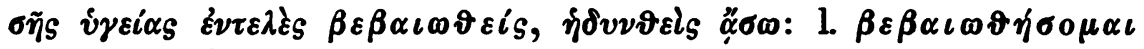

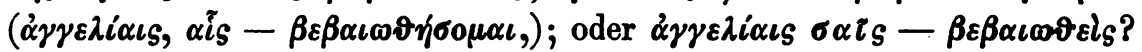

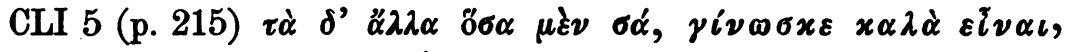

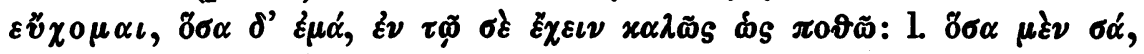
$\gamma i v \omega \sigma x \varepsilon, x \alpha \lambda \dot{\alpha} \varepsilon i v \alpha \iota \varepsilon v ้ \chi 0 \mu \alpha \iota$ etc.

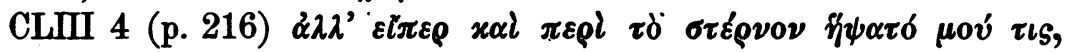

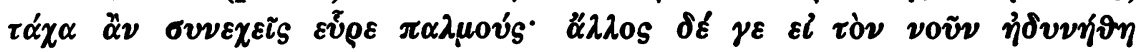

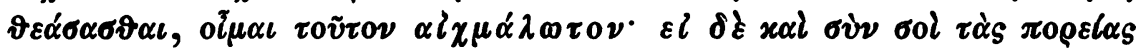

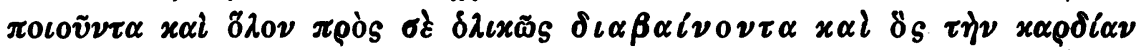

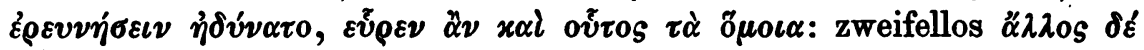

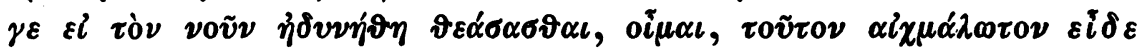

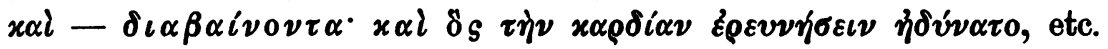

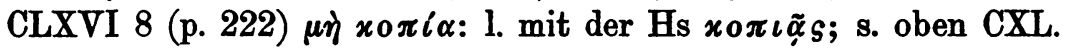

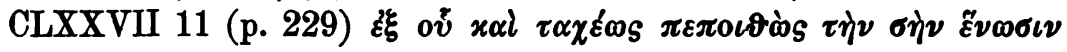

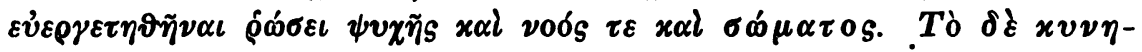

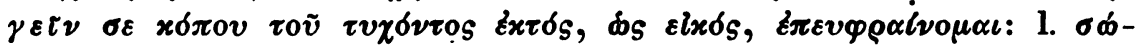
$\mu \alpha \tau 0 s, \tau \grave{o} \delta \dot{\varepsilon} x v \nu \eta \gamma \varepsilon \tilde{\imath} \nu$ etc.

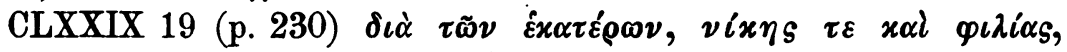

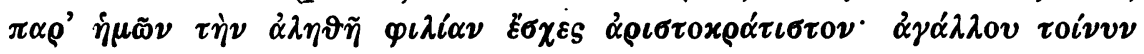

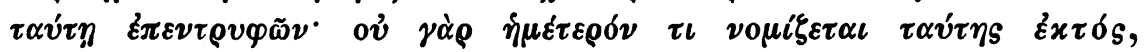

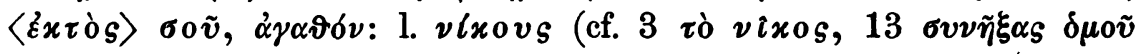

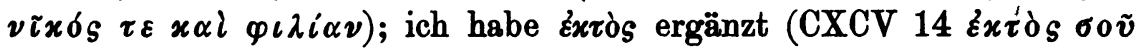

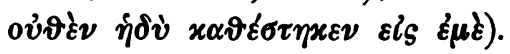

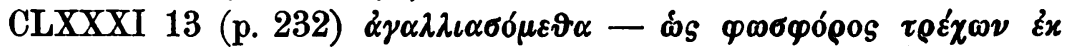

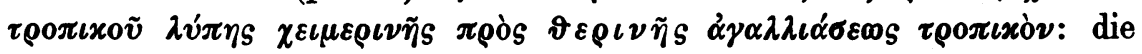

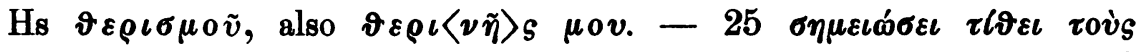

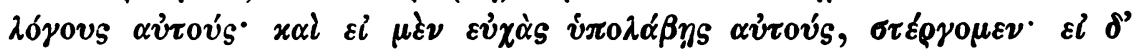

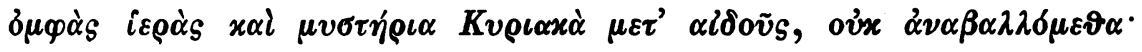




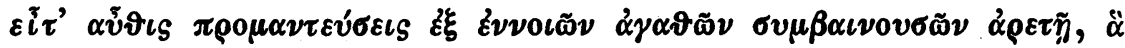

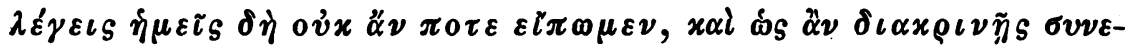

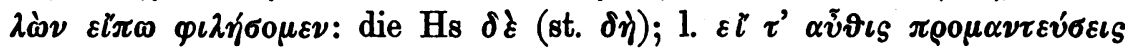

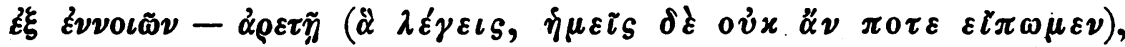

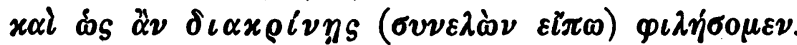

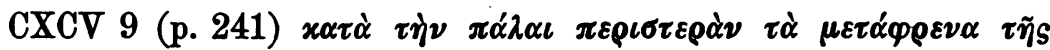

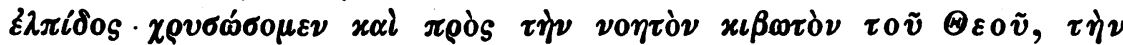

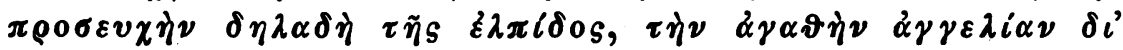

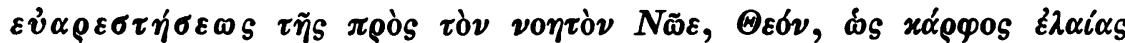

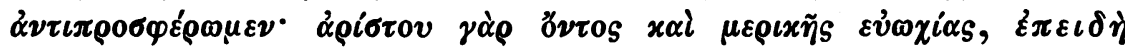

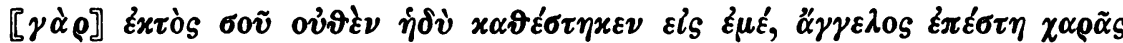

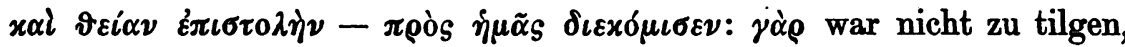

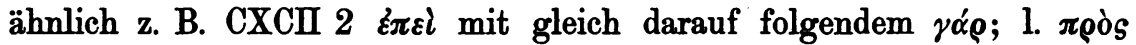

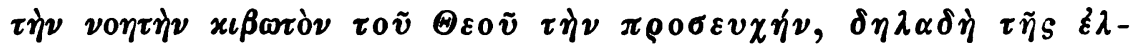
$\pi i \delta 0 s \tau \dot{\eta} \nu \dot{\alpha} \gamma \alpha \boldsymbol{\vartheta} \dot{\eta} \nu \dot{\alpha} \gamma \gamma \varepsilon \lambda i \alpha \nu, \delta i \quad \varepsilon \dot{v} \alpha \rho \varepsilon \sigma \tau \dot{\eta} \sigma \varepsilon \omega s$ etc.

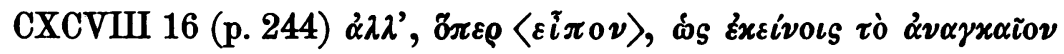

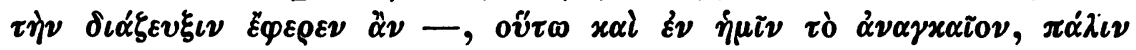

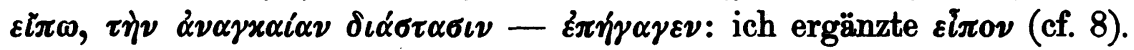

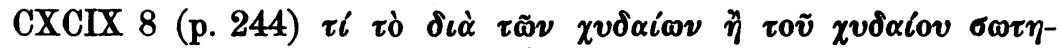

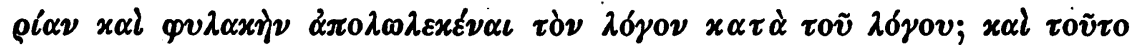

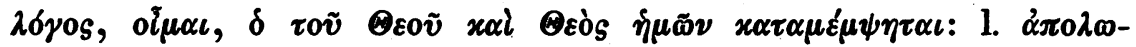

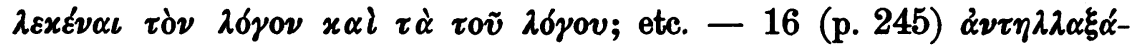

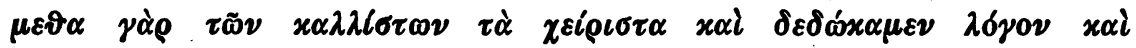

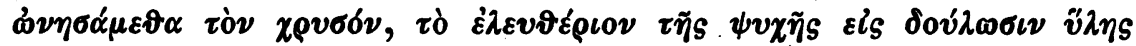

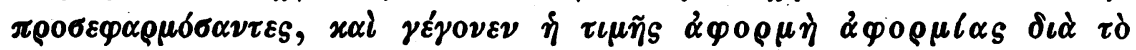

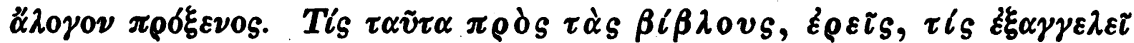

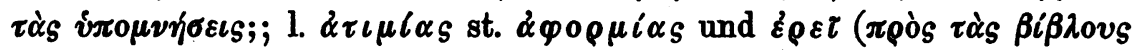

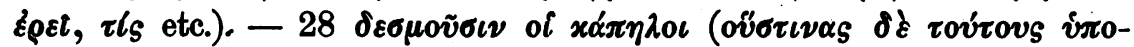

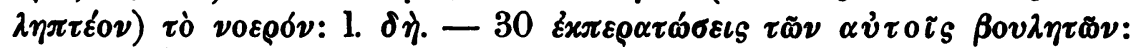

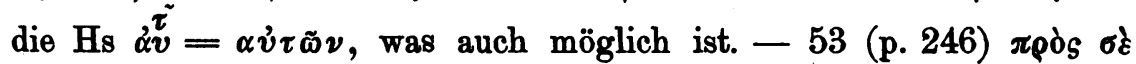

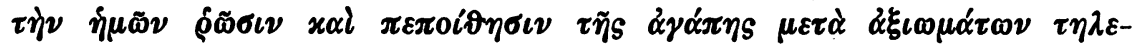

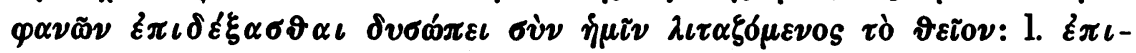
$\delta \varepsilon i \xi \alpha \sigma \vartheta \alpha \iota$.

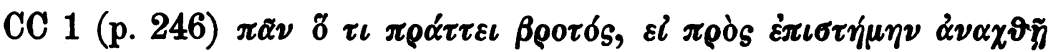

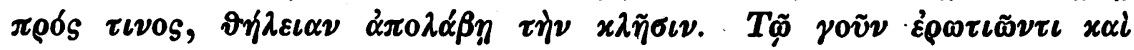

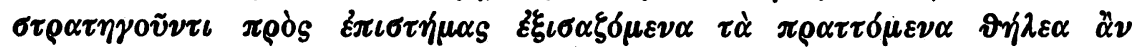

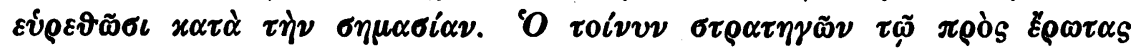

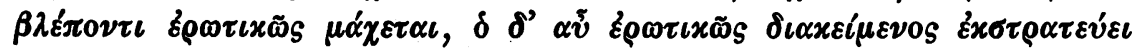

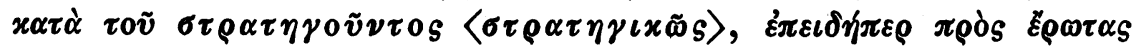

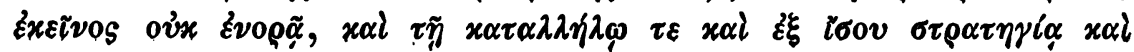




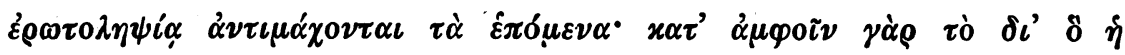

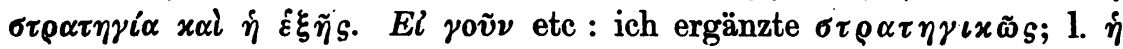

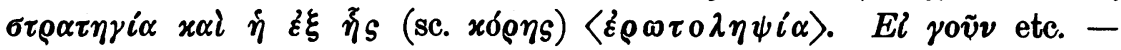

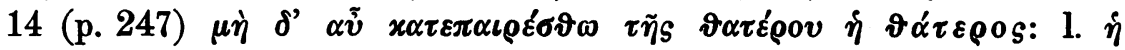

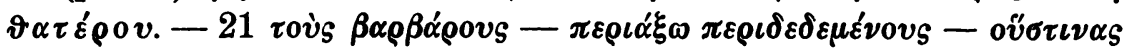

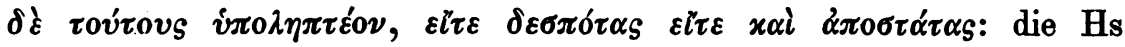
$\delta \varepsilon \tilde{\imath}, 1 . \delta \dot{\eta}$.

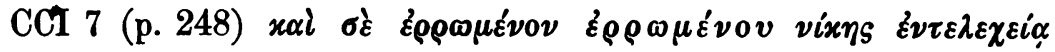

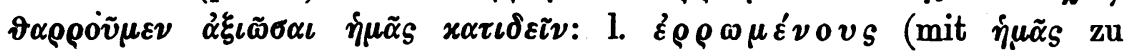
verbinden).

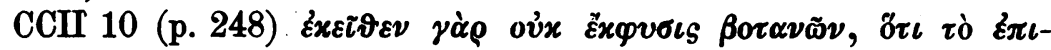

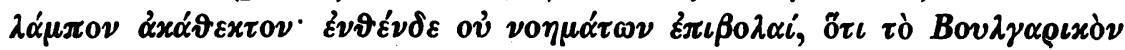

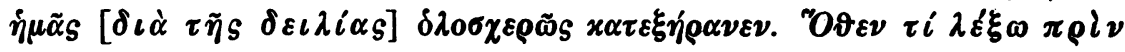

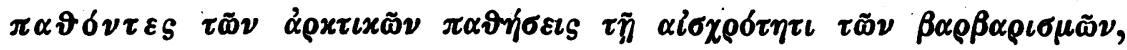

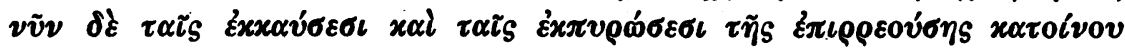

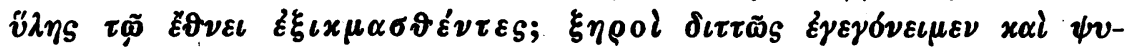

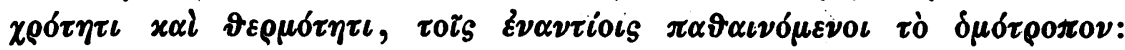
Festa „verba $\delta \iota \dot{\alpha} \tau \tilde{\eta} s \delta \varepsilon \iota \lambda i \alpha s$ valde incerta“; ich schlage $\delta \iota \dot{\alpha} \tau \tilde{\eta} s \beta \alpha \rho-$

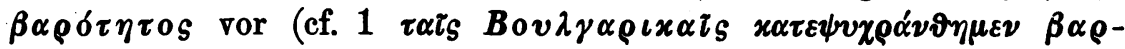

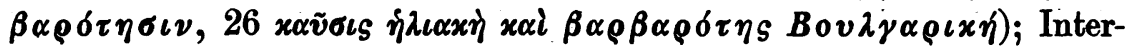
rogationszeichen nach $\xi_{\xi}^{\xi} \iota x \mu \alpha \sigma \vartheta \varepsilon \dot{\varepsilon} \tau \varepsilon$ ist zu tilgen und nach $\lambda \varepsilon^{\prime} \xi \omega \mathrm{zu}$

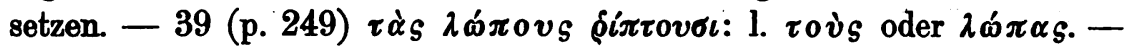

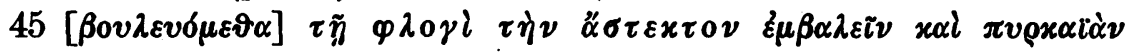

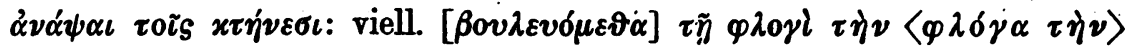

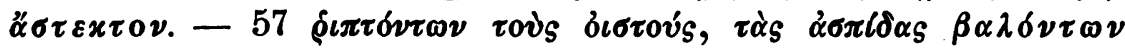

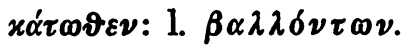

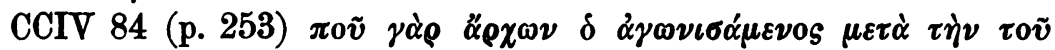

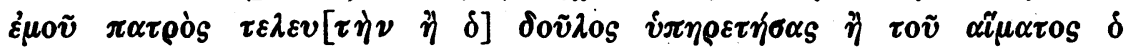

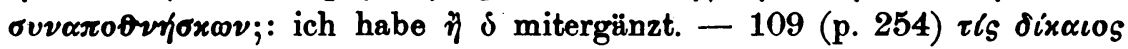

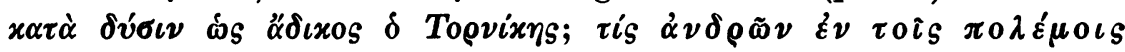

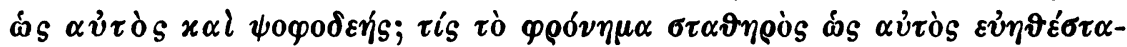

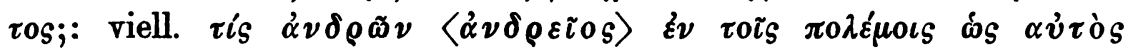
$\langle\delta \varepsilon \iota \lambda \dot{\delta} s\rangle x \alpha \downarrow \psi o \varphi \circ \delta \varepsilon \eta^{\prime} ;$

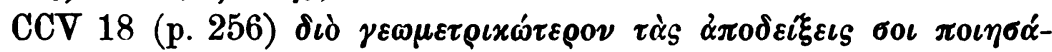

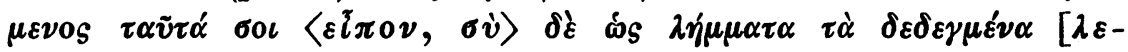

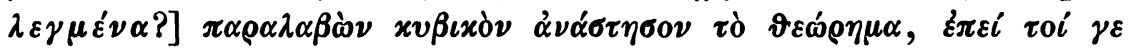

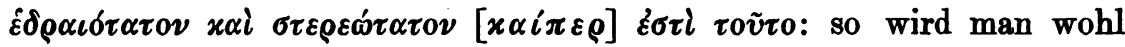

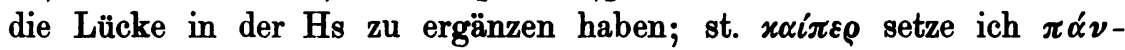
$\tau \omega \nu$ ein.

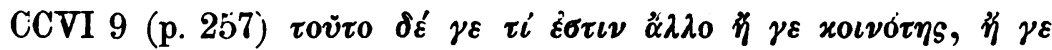

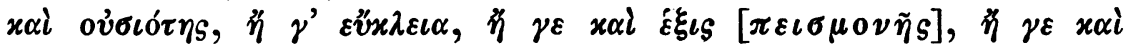




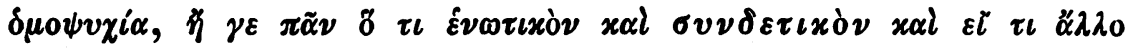

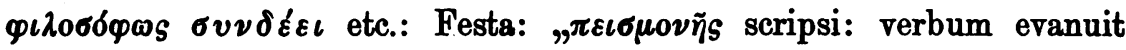
in codice, sed olim $\pi \varepsilon \iota \sigma \mu o ̀ s$ mihi videbar legere"; viell. $\delta \varepsilon \sigma \mu \delta s$. -

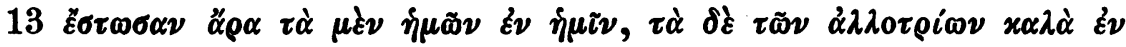

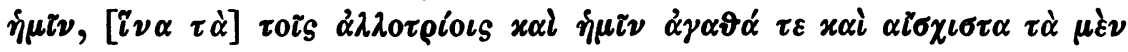

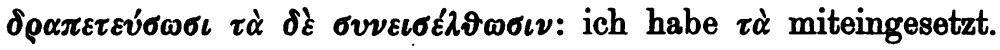

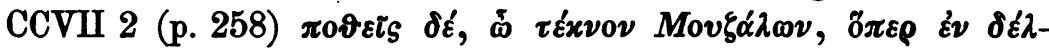

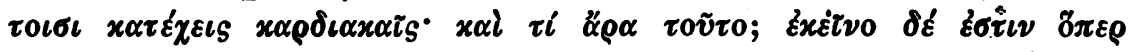

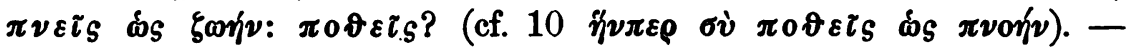

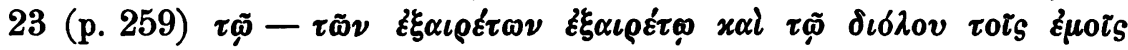

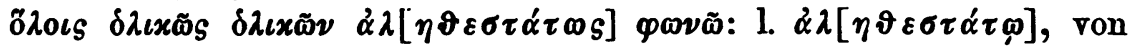
welchem Superl. $\delta \lambda \iota x \tilde{\omega} \nu$ abhängt; $\alpha \lambda \eta \vartheta \varepsilon \sigma \tau \alpha \dot{\alpha} \tau$ ø verlangt übrigens auch der Artikel $\tau \tilde{\omega} \tilde{j}$ vor $\delta \iota 0 ́ \lambda o v$.

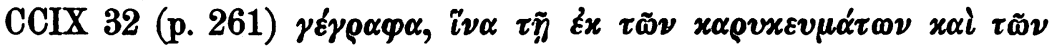

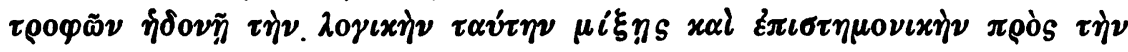

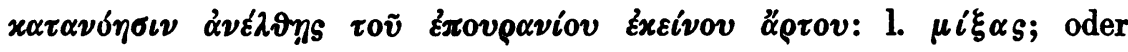
$\dot{\varepsilon} \pi \iota \sigma \tau \eta \mu 0 \nu \iota x \grave{\eta} \nu\langle x \alpha i\rangle \pi \rho \grave{s}$ etc.?

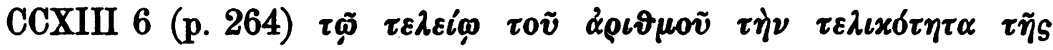

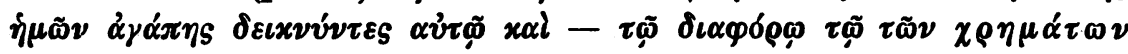

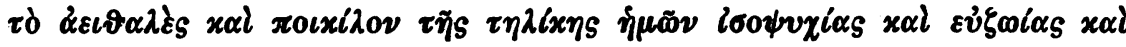

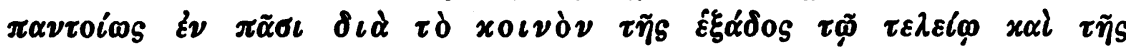

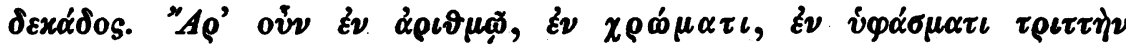

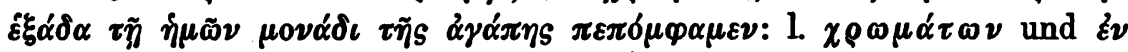

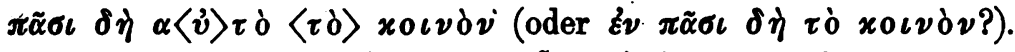

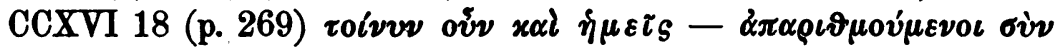

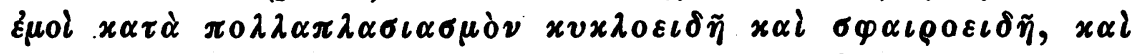

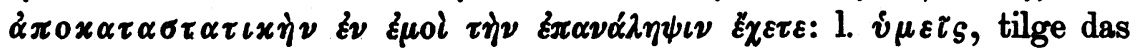
Komma nach $\sigma \varphi \alpha \iota \rho \circ \varepsilon \iota \delta \tilde{\eta}$ und setze nach $\pi 0 \lambda \lambda \alpha \pi \lambda \alpha \sigma \iota \alpha \sigma \mu \delta \nu$ (cf. 10-12).

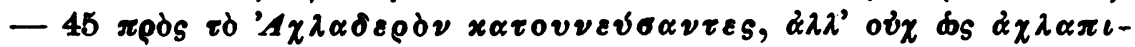

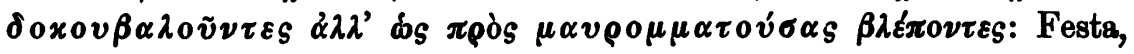
der $x \alpha \tau \alpha \nu \varepsilon v \sigma \alpha \nu \tau \varepsilon S$ schrieb, bemerkt: „cetera non intellego“; $\alpha \chi \lambda \alpha$ -

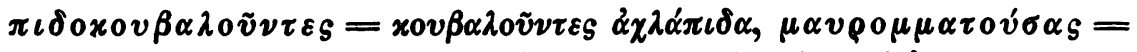

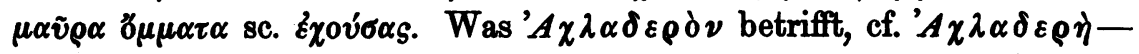
'A $\chi \rho \delta \alpha^{\prime} \eta S$ in meinen Unedierten Inschriften von Mytilene (Leipzig 1900) S. 24.

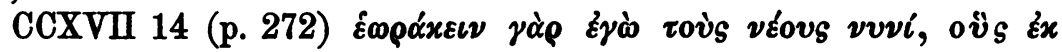

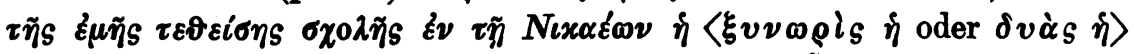

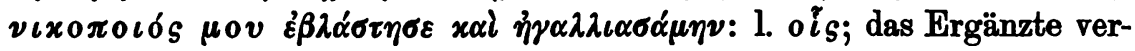

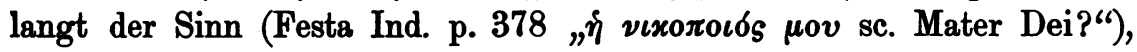

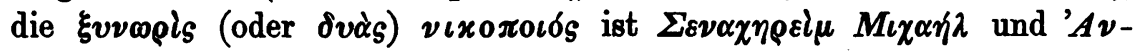

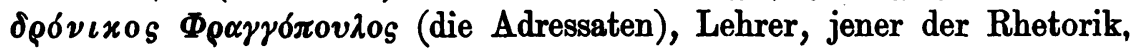




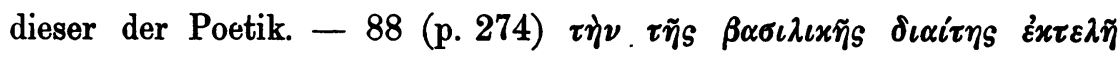

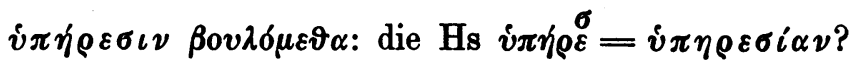

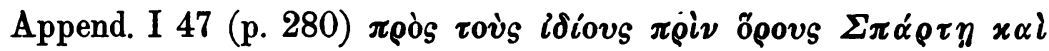

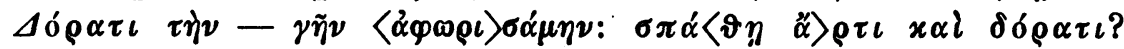
( $\sigma \pi \alpha \dot{\vartheta} \eta$ und $\delta \delta \rho \alpha \tau \iota$ auch Papadopulos).

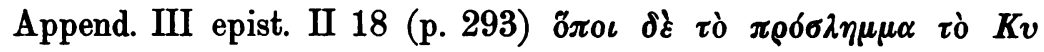

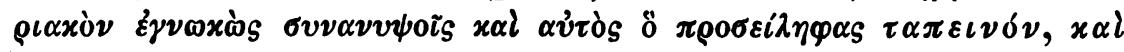

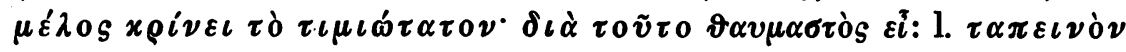

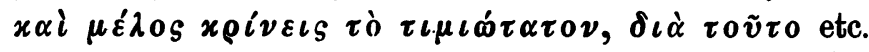

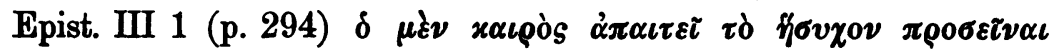

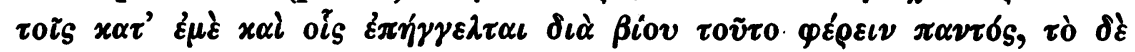

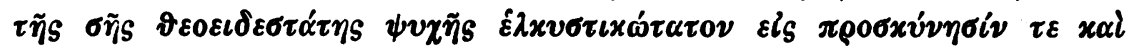

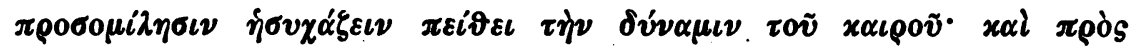

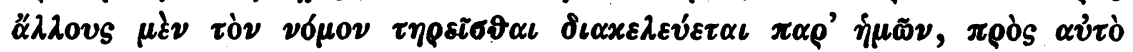

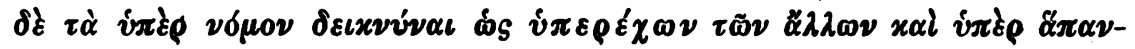

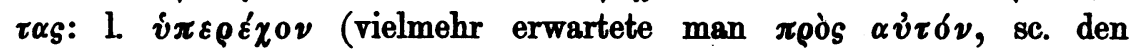
Adressaten, und $\dot{v} \pi \varepsilon \rho \varepsilon \varepsilon \chi 0 \nu \tau \alpha)$.

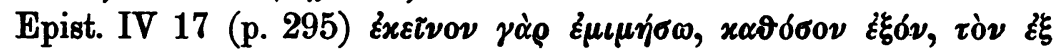

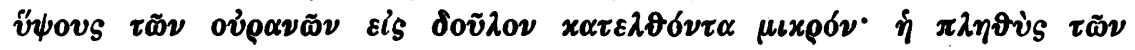

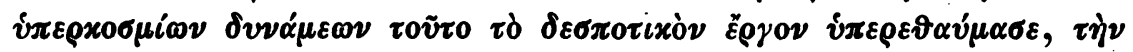

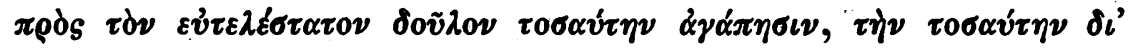

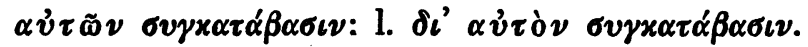

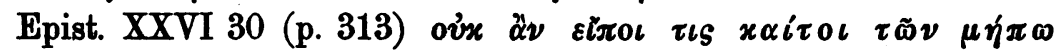

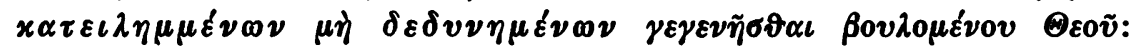
ich lese $\tau \iota \varsigma x a i \tau \iota-\mu \eta \dot{\delta} \delta \delta v \nu \eta \mu \varepsilon v 0 \nu$ (dies mit Festa).

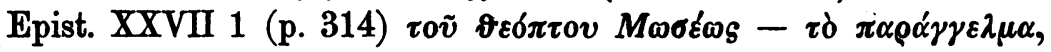

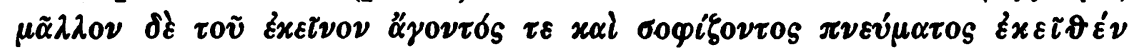

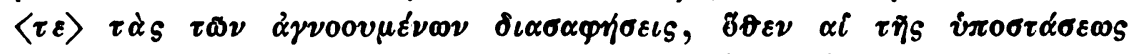

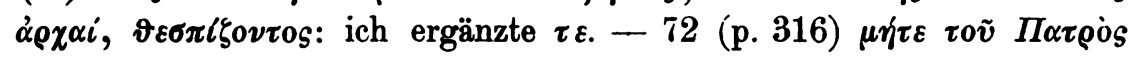

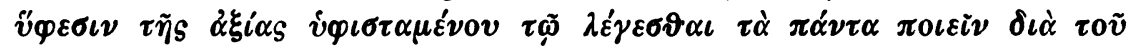

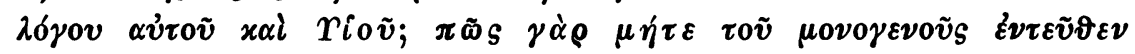

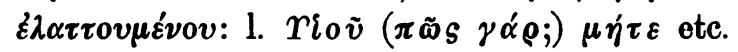

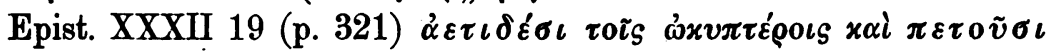

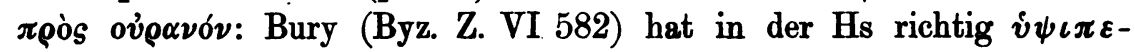
$\tau 0 \tilde{v} \sigma \iota$ (Festa $\pi \alpha \tau \sigma \tilde{v} \sigma \iota$ ) gelesen (und $\dot{\alpha} \varepsilon \tau \iota \delta \varepsilon \tilde{v} \sigma \iota$ verbessert) = Festa Addenda p. XI; die Vermutung $\pi \varepsilon \tau \tilde{\omega} \sigma \iota$ (Papadopulos) ist also überflüssig.

B. - Korrupt sind die Stellen: XIII 3 p. 17 (ö $\tau \iota \delta \xi-\mu \eta े \delta v v \alpha ́ \mu \varepsilon v o s ;$

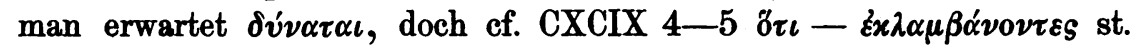

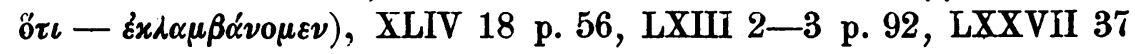
p. 104, LXXXVII 60 p. 115, CIX 16 p. 151 etc. etc. - Eine sichere 
Heilung vermag ich gegenwärtig an diesen Verderbnissen nicht anzuwenden; in manchen Fällen wird man vielleicht mit Textlücken in den betreffenden Hss zu thun haben; auch die Vermutung, Festa könne sich in der Entzifferung mancher Lesarten geirrt haben, bleibt natürlich nicht ausgeschlossen. - Verkehrt ist die Interpunktion auch an vielen

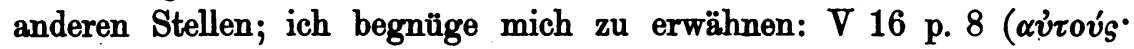

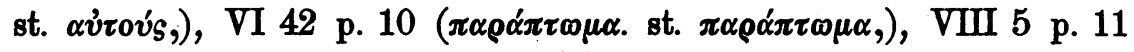

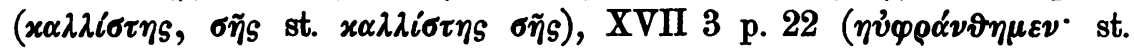
$\eta \dot{ }$

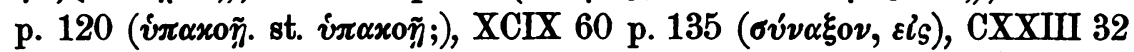

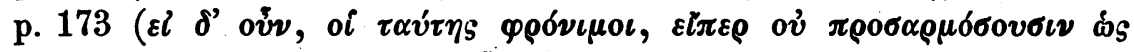

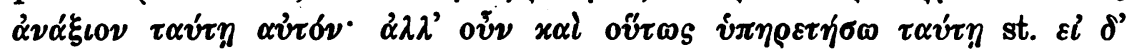

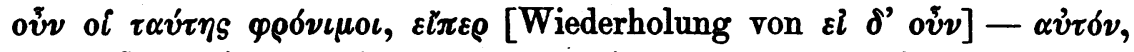

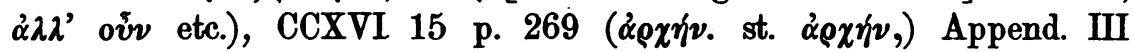

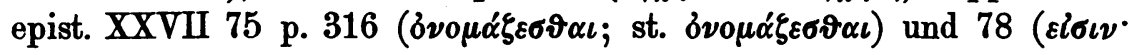
st. $\varepsilon i \sigma \iota \nu$,$) etc. etc. Leicht lälst sich der Eindruck gewinnen, der Heraus-$ geber habe sich allzu sehr an der handschriftlichen Überlieferung festgehalten; die Interpunktion aber der byzantinischen Kopisten ist keine Interpunktion.

C. - Aus vielen Gründen überflüssig scheinen mir die vom Herausgeber an folgenden Stellen entweder faktisch angewandten oder auch blofs vorgeschlagenen Änderungen: XX 16 p. 26, XXVI 5 p. 36, XXVII 28 p. 37 (

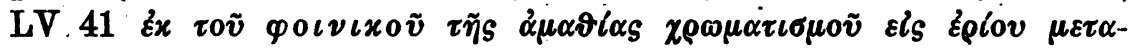
$\left.\beta \lambda \eta \vartheta \varepsilon i_{S}-x \alpha \vartheta \alpha \rho \sigma \tau \eta \tau \alpha\right)$, XXXII 28 p. 42, XXXV 5 p. 43, XXXVI 34 p. 45, XXXIX 21 p. 50 , XLIV 11 p. 56 und 39 p. $57=$ Addenda

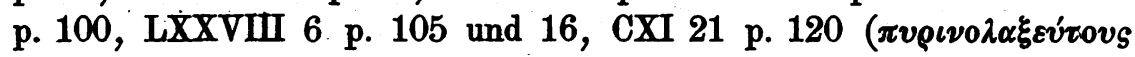

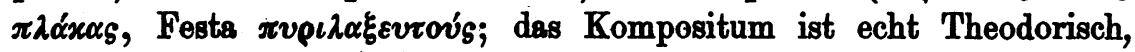
keineswegs seltsamer als viele Monstra des kaiserlichen Geistes), XCIV 9

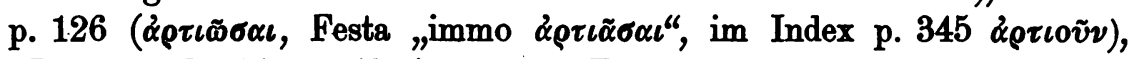

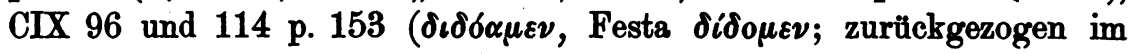
Indiculus p. 414), CXIII 21 p. 57 (zurück in den Addenda), CXVI 25

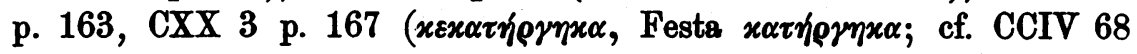

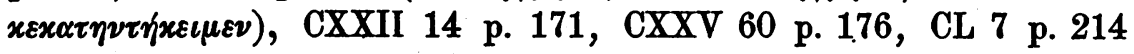
(memoriae lapsus in Zitaten sind nicht selten), CXXXII 6 p. 186,

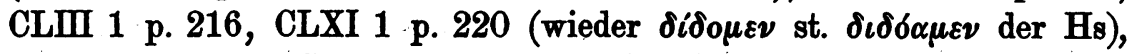

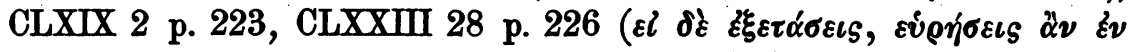

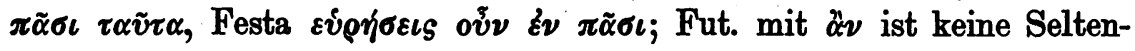

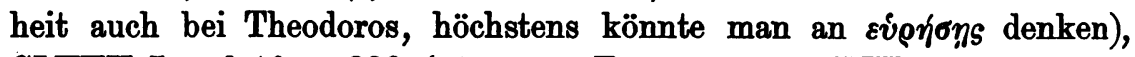

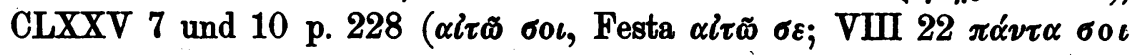

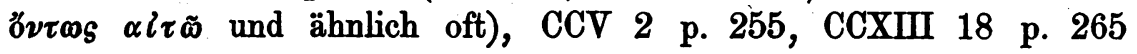




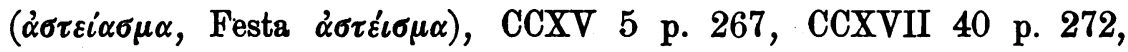

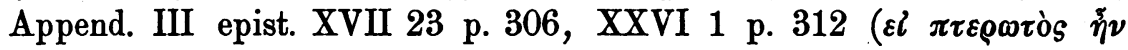

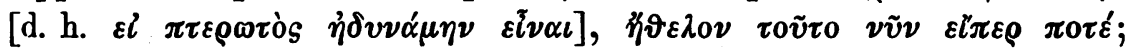

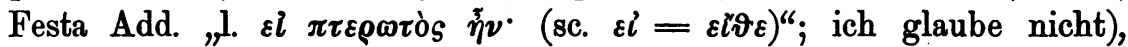

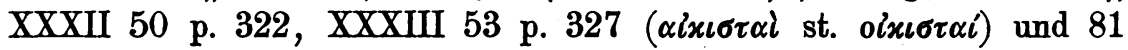
p. 328, Append. IV 53 und 60 ( $\tau \varepsilon \lambda \mu \alpha \tau i \alpha \iota$, Festa $\tau \varepsilon \lambda \mu \alpha \tau \iota \alpha i \tilde{o l}$; cf. $\tau 0 \lambda-$

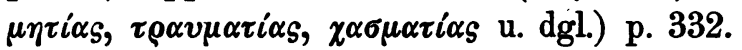

D. - Was des Kaisers sprachliche Einkleidung seiner eben nicht sehr klaren Gedanken und die Benutzung des reichen Wortschatzes (darüber ein anderes Mal) betrifft, so sehen wir uns eine Erscheinung entgegentreten, welche auf dem ganzen Gebiete der byzantinischen Litteratur zweifellos einzig und allein dasteht. Am eigentümlichsten ist bei Theodoros die Syntax, ein wahres litterarisches Unikum seine collocatio verborum; sie hängt offenbar mit dem ganzen Charakter des Mannes eng zusammen, und „auf seinen Charakter . . ist . . von . . starkem Einflufs .. sein körperlicher Zustand ... geblieben" (Heisenberg a. a. 0 . p. 213). Zwar ist es recht interessant, die häufig, oft auch hübsch, erhobenen Klagen des Kaisers über seine Krankheit (Epilepsie) zu lesen und die Verspottungen der kaiserlichen Ärzte in ihrer leihe zu

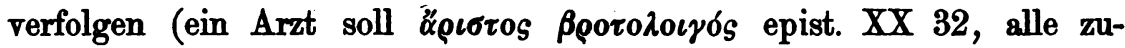

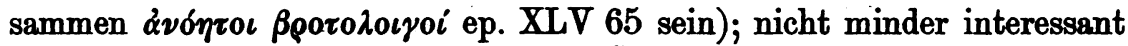
ist es aber, die Ungeschicktheit in der Äufserung der Gedanken von dem

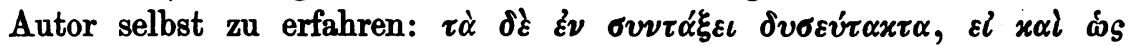

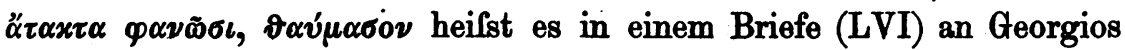
Akropolites, und gleich darauf wird uns der zerreifsende Wortschnörkel

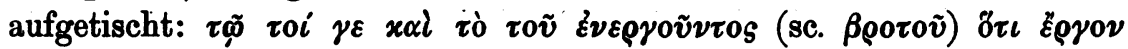

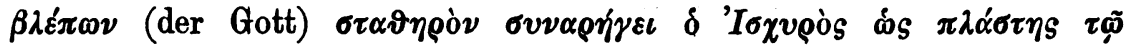

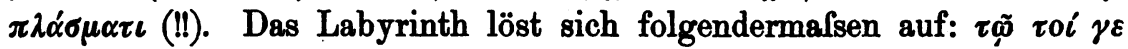

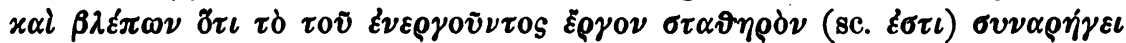

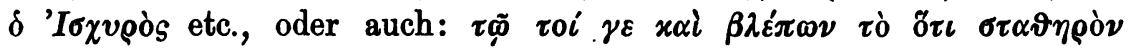

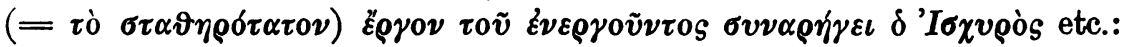
wie es dem Kaiser beliebig gewesen, dies wüfste uns er allein zu sagen. Zwar hat Festa schlechtweg an ä $\tau \varepsilon$ (st. ö $\tau \iota$ ) oder an $\beta \lambda \varepsilon \pi \varepsilon \iota$ (st. $\beta \lambda \varepsilon j \omega \nu$ ) gedacht; mit wie viel Recht, davon legen ein unstreitbares Zeugnis zahlreiche Stellen ab, von denen ich unten eine kleine Auslese

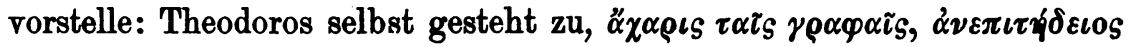

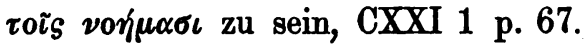

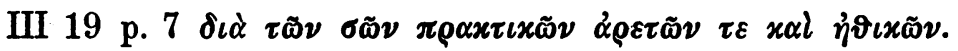

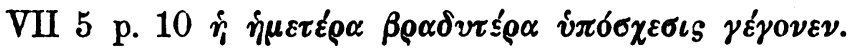

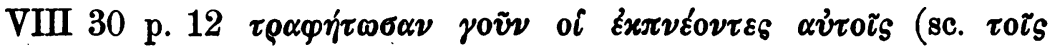




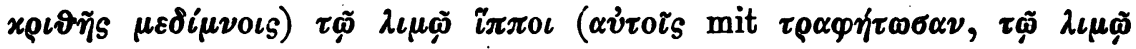
mit $\varepsilon x \pi \nu \varepsilon 0 \nu \tau \varepsilon \xi !)$.

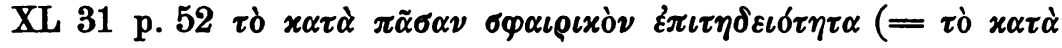

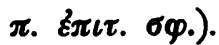

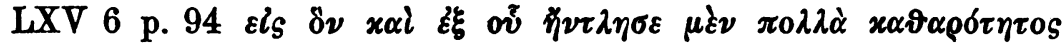

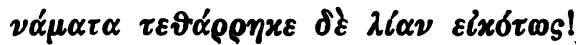

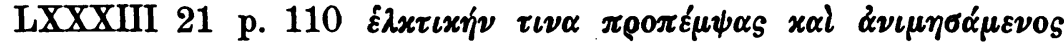

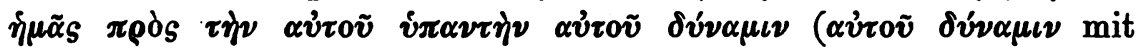

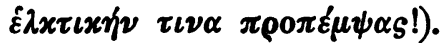

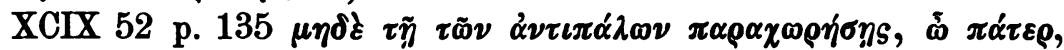

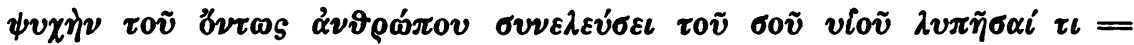

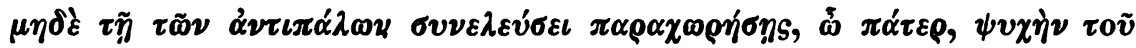

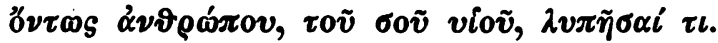

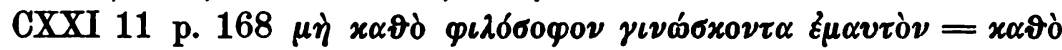

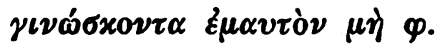

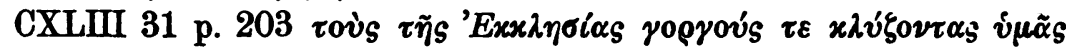

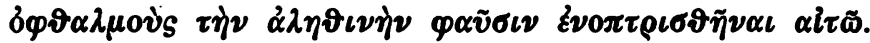

Offenbar hat man es nicht mehr mit einer einfachen "collocatio verborum artificiosior" zu thun, und ich mufs bedauern, dafs der Herausgeber der in den Hss überlieferten Wortstellung sehr oft zu Leib gegangen ist, gewifs nicht in der Absicht des seltsamen Autors.

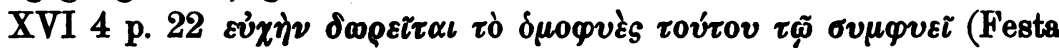

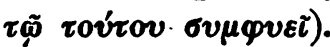

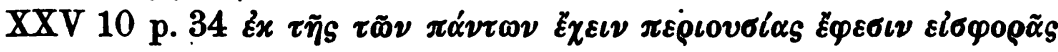

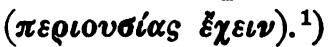

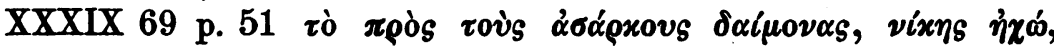

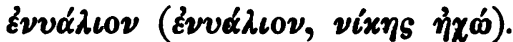

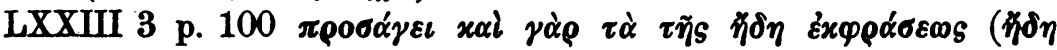

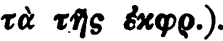

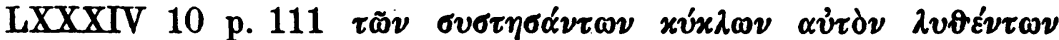

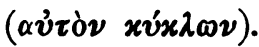

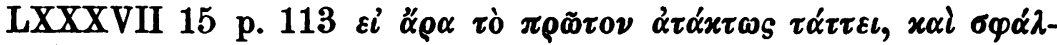

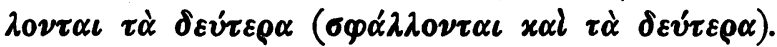

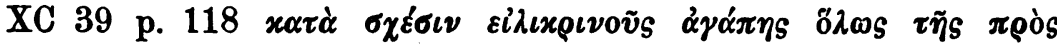

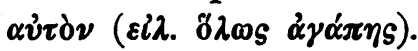

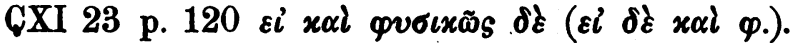

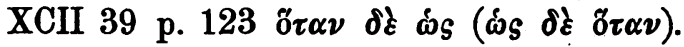

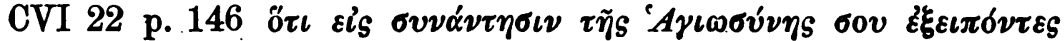

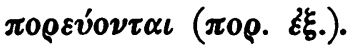

1) Eingeklammert habe ich überall die Metathesen des Herausgebers. 


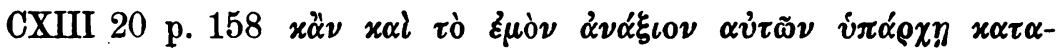

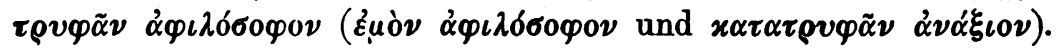

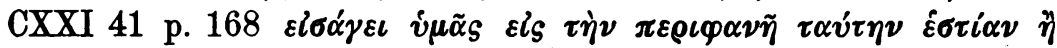

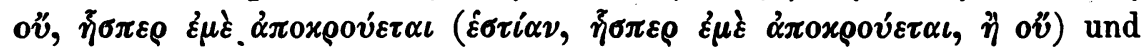

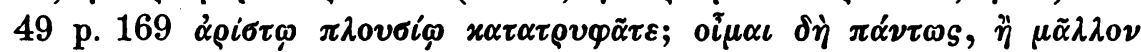

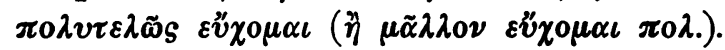

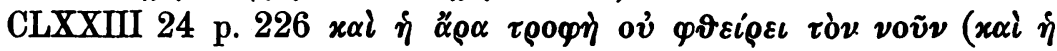
$\tau \varrho \circ \varphi \eta े \ddot{\alpha} \alpha)$.

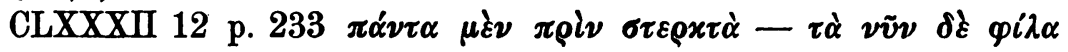
$(\pi \rho i v \mu \dot{v} v)$.

Etc. etc.

E. - Dagegen schwerlich zu rechtfertigen ist der Konservatismus

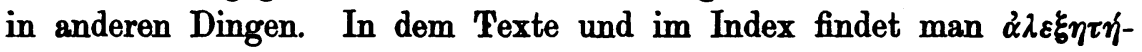

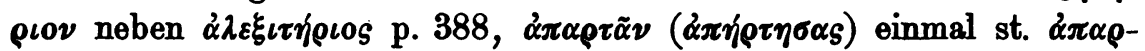

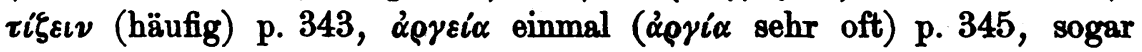
LXXXVIII $4 \pi \rho \alpha \gamma \mu \alpha \tau \iota \tilde{\nu}$ (auch im Index p. 387) st. des häufigen $\pi \rho \alpha \gamma \mu \alpha \tau \varepsilon i \alpha$, lauter wertlose Iotazismen-Schreibungen (cf. auch XCIC 79

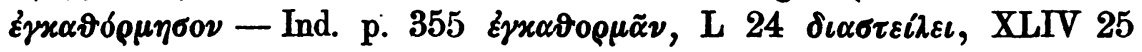

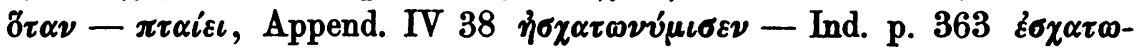

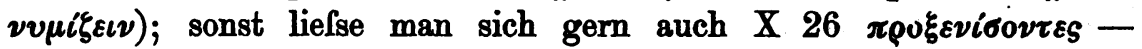

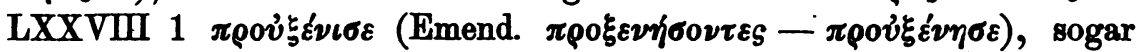

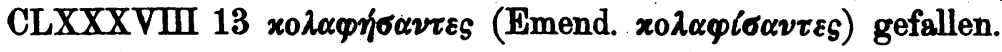

XIV $21 \pi \varepsilon \rho \iota x \alpha \lambda v \dot{v} \alpha \alpha_{S}-\pi \varepsilon \rho \iota \varepsilon x \alpha \dot{\lambda} v \nu \alpha \alpha_{S}$ („aor. $\varepsilon x \alpha \dot{\lambda} \lambda v \nu \alpha$ fictum videtur

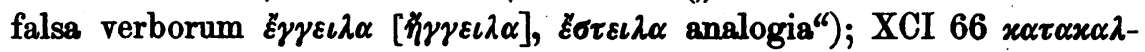
$\lambda v^{\prime} \alpha_{s}$ (Cod. $x \alpha \tau \alpha x \alpha \lambda v_{v \alpha s}$ ), 60 das sichere $x \alpha \tau \alpha x \alpha \lambda \lambda v \nu \varepsilon \tilde{S}$ (Cod. $x \alpha \tau \alpha-$

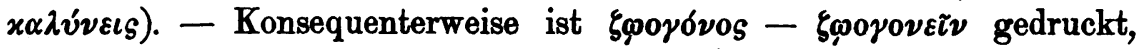

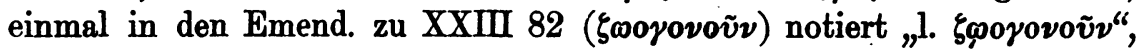
dagegen $\xi \omega \gamma \rho \alpha \dot{\varphi} \eta \mu \alpha-\xi \omega \gamma \rho \alpha \varphi \eta \mu \alpha \tau \alpha$, wieder XXXVI $73 \lambda \eta \rho \oplus \delta \eta \mu \alpha \tau \alpha$

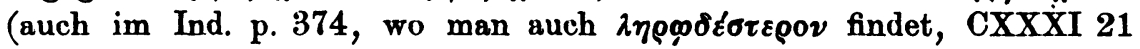

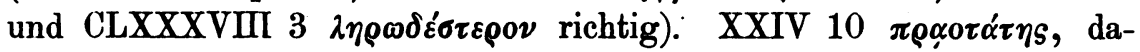

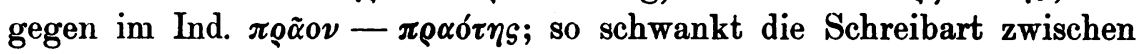
$\sigma \omega^{\prime} \xi \varepsilon \iota \nu$ ( $\dot{\varepsilon} \pi \alpha \nu \alpha \sigma \omega^{\prime} \xi \varepsilon \tau \alpha \iota$ im Texte, $\dot{\varepsilon} \pi \alpha \nu \alpha \sigma \omega^{\prime} \xi \sigma \sigma \alpha \iota$ im Ind.) und $\sigma \dot{\omega} \xi \varepsilon \nu$, etc.

F. - Falsch sind im Index: p. $339 \dot{\alpha} \mu \varphi i o \nu$ (im Texte $\dot{\alpha} \mu \varphi i \omega \nu$ ), $341 \dot{\alpha} \nu \vartheta 0 \sigma \mu i o v$ (im Texte $\dot{\alpha} \nu \vartheta 0 \sigma \mu i o v$; notiert in Viz. Vrem.), $\dot{\alpha} \sigma x \lambda \eta-$

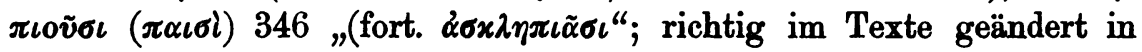

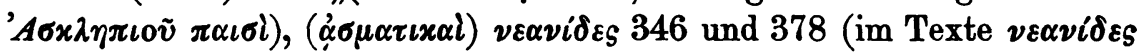

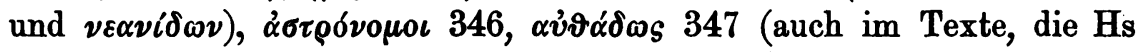

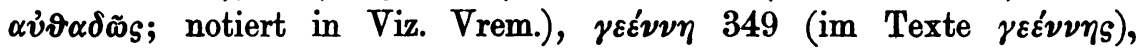

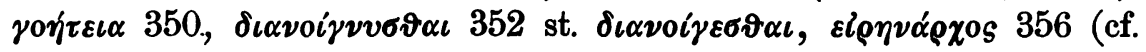

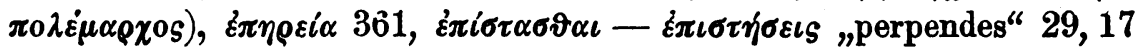

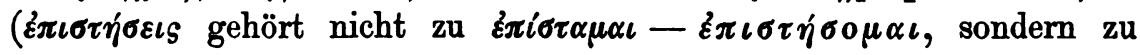




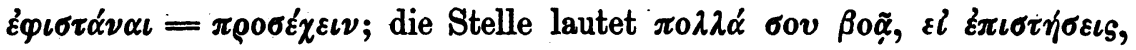

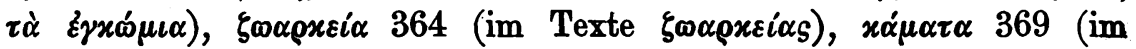
Texte $x \alpha \mu \alpha ́ \tau \omega \nu$ ), $x \alpha \tau \varepsilon v o \delta \varepsilon \tilde{\sigma \vartheta \alpha \iota} 370$ (im Texte $x \alpha \tau \varepsilon v o \delta o \tilde{v}$; notiert im

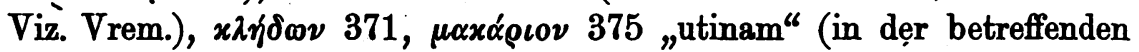

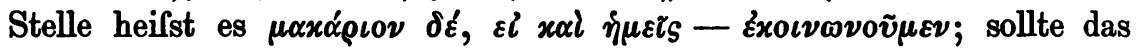

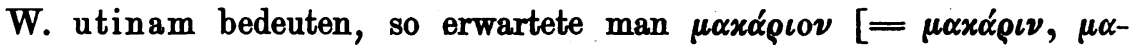

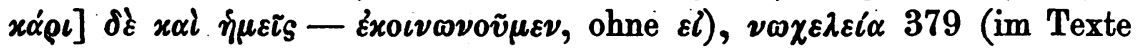

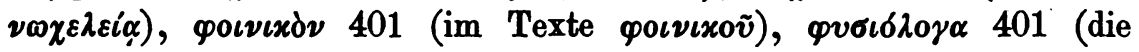

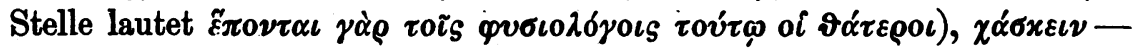

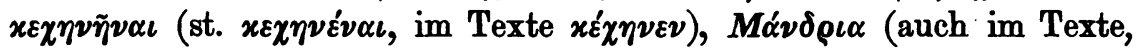

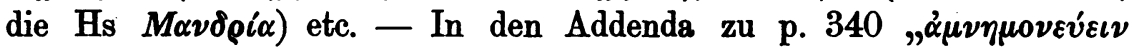

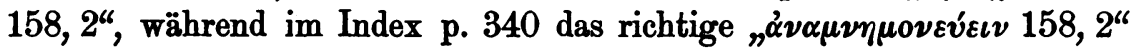

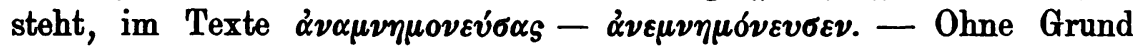

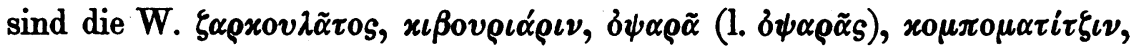

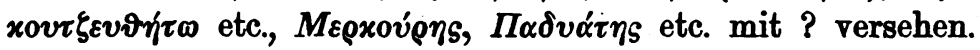

G. - Die Ausgabe ist leider auch durch Druckfehler entstellt; viele sind in den Emendanda berichtigt, ich habe gegen 80 gefunden,

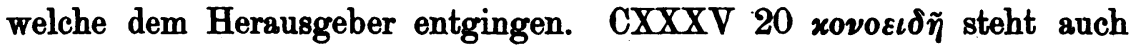

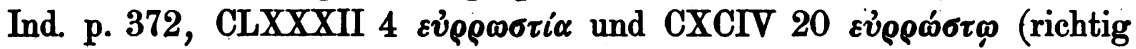

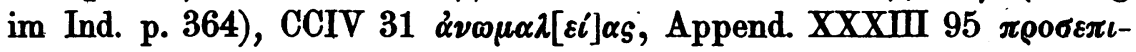

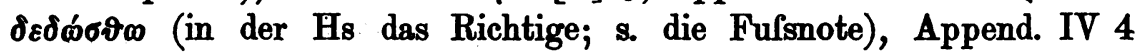

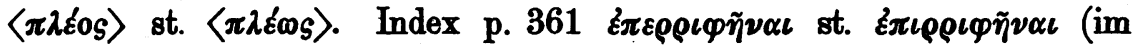

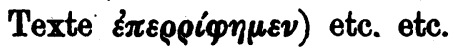

Mit meinen Emendationen und Bemerkungen habe ich weder den Wert der Publikation noch das Verdienst des Herausgebers abschwächen wollen; sollten dieselben vom Herrn Verfasser zum Nutzen einer zweiten, notwendigen Ausgabe verwendet werden, so würde meine Besprechung der Princeps das Ziel erreicht haben.

Saloniki.

P. N. Papageorgin. 